\title{
Effects of 3-D thermal radiation on the development of a shallow cumulus cloud field
}

\author{
Carolin Klinger $^{1,2}$, Bernhard Mayer ${ }^{1}$, Fabian Jakub ${ }^{1}$, Tobias Zinner ${ }^{1}$, Seung-Bu Park ${ }^{3}$, and Pierre Gentine ${ }^{3}$ \\ ${ }^{1}$ Ludwig Maximilians-Universität München, Fakultät für Physik, Meteorologisches Institut München, Munich, Germany \\ ${ }^{2}$ currently at: Chemical Sciences Division, NOAA Earth System Research Laboratory (ESRL), Boulder, CO, USA \\ ${ }^{3}$ Department of Earth and Environmental Engineering, Earth Institute, Columbia University, New York, NY, USA
}

Correspondence to: Carolin Klinger (carolin.klinger@physik.uni-muenchen.de)

Received: 7 October 2016 - Discussion started: 11 November 2016

Revised: 25 March 2017 - Accepted: 27 March 2017 - Published: 28 April 2017

\begin{abstract}
We investigate the effects of thermal radiation on cloud development in large-eddy simulations (LESs) with the UCLA-LES model. We investigate single convective clouds (driven by a warm bubble) at $50 \mathrm{~m}$ horizontal resolution and a large cumulus cloud field at 50 and $100 \mathrm{~m}$ horizontal resolutions. We compare the newly developed 3-D Neighboring Column Approximation with the independent column approximation and a simulation without radiation and their respective impact on clouds. Thermal radiation causes strong local cooling at cloud tops accompanied by a modest warming at the cloud bottom and, in the case of the 3-D scheme, also cloud side cooling. 3-D thermal radiation causes systematically larger cooling when averaged over the model domain. In order to investigate the effects of local cooling on the clouds and to separate these local effects from a systematically larger cooling effect in the modeling domain, we apply the radiative transfer solutions in different ways. The direct effect of heating and cooling at the clouds is applied (local thermal radiation) in a first simulation. Furthermore, a horizontal average of the 1-D and 3-D radiation in each layer is used to study the effect of local cloud radiation as opposed to the domain-averaged effect. These averaged radiation simulations exhibit a cooling profile with stronger cooling in the cloudy layers. In a final setup, we replace the radiation simulation by a uniform cooling of $2.6 \mathrm{~K} \mathrm{day}^{-1}$. To focus on the radiation effects themselves and to avoid possible feedbacks, we fixed surface fluxes of latent and sensible heat and omitted the formation of rain in our simulations.
\end{abstract}

Local thermal radiation changes cloud circulation in the single cloud simulations, as well as in the shallow cumulus cloud field, by causing stronger updrafts and stronger subsiding shells. In our cumulus cloud field simulation, we find that local radiation enhances the circulation compared to the averaged radiation applications. In addition, we find that thermal radiation triggers the organization of clouds in two different ways. First, local interactive radiation leads to the formation of cell structures; later on, larger clouds develop. Comparing the effects of 3-D and 1-D thermal radiation, we find that organization effects of 3-D local thermal radiation are usually stronger than the 1-D counterpart. Horizontally averaged radiation causes more clouds and deeper clouds than a no radiation simulation but, in general less-organized clouds than in the local radiation simulations. Applying a constant cooling to the simulations leads to a similar development of the cloud field as in the case of averaged radiation, but less water condenses overall in the simulation. Generally, clouds contain more liquid water if radiation is accounted for. Furthermore, thermal radiation enhances turbulence and mixing as well as the size and lifetime of clouds. Local thermal radiation produces larger clouds with longer lifetimes.

The cloud fields in the 100 and $50 \mathrm{~m}$ resolution simulations develop similarly; however, 3-D local effects are stronger in the $100 \mathrm{~m}$ simulations which might indicate a limit of our 3-D radiation parameterization. 


\section{Introduction}

Clouds are a key element for accurate climate and weather prediction and cause large uncertainties in the prediction of both (Boucher et al., 2013). Clouds play an important, yet poorly quantified, role in climate change. Key questions arising from the limited understanding of clouds in climate prediction were recently pointed out by Bony et al. (2015). These questions include the role of cloud organization or the role of cloud convection in a changing climate. Feedbacks of radiative effects on cloud dynamics and microphysics are one component which modify cloud development, and limited understanding of these processes contributes to the uncertainty in climate prediction.

Solar and thermal radiation drive weather and climate and affect cloud formation. Different studies in the past looked at radiative effects caused in clouds. Thermal cooling rates in stratiform clouds were found to vary between 2 and $40 \mathrm{~K} \mathrm{~h}^{-1}$ (Feigelson, 1973, 1984; Davies and Alves, 1989; Stephens, 1978). These studies showed that thermal heating and cooling rates depend, e.g., on the liquid water content of a cloud (Feigelson, 1973, 1984; Davies and Alves, 1989; Lábó and Geresdi, 2016) and on the strength of the inversion layer above the clouds (Twomey, 1983). Harshvardhan et al. (1981) was one of the first to look at 3-D effects on clouds by applying radiative transfer to a cuboid cloud. They found strong cooling on the sides of the cuboid cloud, causing up to a factor of 3 difference in the cooling rate when comparing their result to a plane-parallel treatment of a cloud. In a follow-up study, Harshvardhan and Weinman (1982) extended this approach to a regular array of cuboid clouds. Again, it was found that 3-D cooling rates can exceed those of 1-D radiative transfer calculations by a factor of 2-3. Depending on the aspect ratio of the cuboid cloud, this factor can be lower or higher. Guan et al. (1995) further investigated those effects with an axially symmetric 3-D radiative transfer model focusing on peaks and holes of isothermal stratiform clouds as well as non-isothermal isolated cylindrical clouds. In holes of stratiform clouds, cooling rates were found to be smaller in 3-D compared to 1-D radiation simulations. The cylindrical clouds showed cooling at cloud top (up to $-34 \mathrm{Kh}^{-1}$ ) and cloud side $\left(-14 \mathrm{~K} \mathrm{~h}^{-1}\right.$ ). Most of the cooling was found within the first $20 \mathrm{~m}$ from the cloud side.

More recent studies using accurate radiative transfer models (e.g., Monte Carlo models) found strong 3-D local thermal cooling rates reaching up to $300-600 \mathrm{~K} \mathrm{day}^{-1}$ (e.g., Kablick et al., 2011; Klinger and Mayer, 2014) in realistic 3-D cloud field simulations. It was shown that 3-D cooling rates exceed 1-D cooling rates both in magnitude and by an additional cloud side cooling. An example of 3-D thermal cooling rates in a cumulus cloud field (calculated on a largeeddy simulation (LES) time snapshot from Cahalan et al., 2005) is shown in Fig. 1.

The figure shows cooling rates at cloud tops and cloud sides, reaching values up to $300 \mathrm{Kday}^{-1}$. In addition, mod-

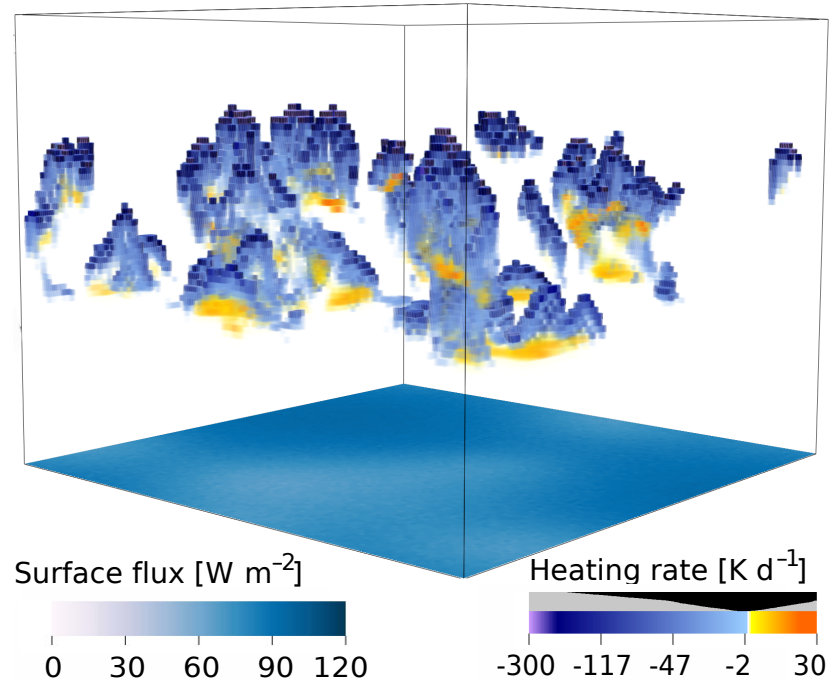

Figure 1. Three-dimensional heating and cooling rates and surface fluxes in a cumulus cloud field, calculated with the Monte Carlo model MYSTIC (Mayer, 2009; Klinger and Mayer, 2014). The black and gray bar shows the opacity of heating rates.

est warming at the cloud bottom (maximum $30 \mathrm{~K} \mathrm{day}^{-1}$ ) is found. The resulting change in the surface flux between cloud-free and cloudy areas is small in the thermal spectral range. The magnitude of these cooling rates suggests that thermal radiation likely has an impact on cloud development (Davies and Alves, 1989).

Solar radiative effects are different from thermal radiative effects. In the solar spectral range, absorption of sunlight at the illuminated cloud sides causes heating rates up to $100 \mathrm{~K} \mathrm{day}^{-1}$ (O'Hirok and Gautier, 2005; Jakub and Mayer, 2015). The location of these heating rates varies with the solar zenith angle. In addition, the surface fluxes vary dramatically between directly illuminated and shaded areas (Wapler and Mayer, 2008; Wissmeier and Buras, 2014; Jakub and Mayer, 2015). Again, the location of the shadow or the directly illuminated surface depends on the solar zenith angle.

The above-mentioned radiative effects are known to affect cloud development. Koračin et al. (1998) coupled a 3-D Monte Carlo model to a 2-D cloud model. They found situations where 3-D solar radiative transfer might become important but did not investigate the solar effects on cloud development any further. Schumann et al. (2002) studied the cloud shadow effect in the solar spectral range in the convective boundary layer with an idealized setup. They showed nonsteady convective motion if the shadow of the cloud was located directly below the cloud and an reduction in the cloud's own buoyancy, compared to a shifted shadow. Cloud shading from the anvils of thunderstorm clouds and the potential feedback on thunderstorm dynamics was investigated in a number of studies. Markowski and Harrington (2005) used a very simplified radiative transfer approach by applying a surface cooling of $6 \mathrm{Kh}^{-1}$ in their simulations. The change 
in surface sensible heat flux led to differences in the strength of the thunderstorm. The effect of cloud shading and the resulting change in surface fluxes were further addressed by Frame et al. (2009); Frame and Markowski (2010, 2013).

Thermal cooling is known to drive the development of stratus clouds. Möller (1951) already stated that cooling at cloud top and warming at the cloud bottom can drive convection in clouds by vertical differential heating and cooling as well as the following destabilization. Therefore, stratus clouds might alter to stratocumulus and altostratus to altocumulus. Similar to Möller (1951), Curry and Herman (1985) found increased convection due to radiation. Furthermore, they found an increased liquid water content and enhanced droplet growth. Destabilization and enhanced turbulence caused by the vertical differential heating and cooling was found in addition by Sommerai (1976), Fu et al. (1995), Petters et al. (2012) and Lilly (1988). Larson et al. (2001) showed that thermal cooling, on the one hand, enhances condensation and thus increases liquid water content; on the other hand, radiation causes more entrainment and therefore a decrease in liquid water content. Fu et al. (1995) found that the clear sky cooling enhances convection and increases precipitation by $5 \%$. Tao et al. (1993) showed an increase in precipitation of $14-31 \%$ due to thermal effects, and Tao et al. (1996) saw an increase in relative humidity of the environment, enhanced circulation and microphysical processes.

In a recent study, Bellon and Geoffroy (2016a) investigated the stratocumulus 1-D radiative effect in a set of equilibrium simulations. It was found that, depending on the sea surface temperature (SST), radiative cooling at cloud top is crucial for the existence of the equilibrium stratocumulus and causes enhanced turbulence by buoyancy production, more entrainment and a deepening of the boundary layer. Based on the results of the first study, Bellon and Geoffroy (2016b) investigated different approximations of the radiative effect, such as column or horizontally averaged radiation. They found that the radiative effect has to be represented in some detail at the cloud top to account for the enhanced turbulence and mixing and therefore determine the existence of the stratocumulus. Other studies also addressed the differences in cloud development caused by local or homogenized cooling in simulations of deep convective clouds. Xu and Randall (1995) found a longer lifetime of clouds when simulating clouds with local radiation. Thermal radiation increased turbulence on short timescales and the cloud development itself on longer timescales.

In a detailed study, Petch and Gray (2001) investigated the robustness of cloud simulations with varying horizontal resolutions ( $2 \mathrm{~km}, 1 \mathrm{~km}, 500 \mathrm{~m})$, domain size, microphysical parameterizations and different radiation schemes. They also compared results from a 2-D cloud model and a 3-D cloud model. In terms of resolution, they found a sensitivity of the mass flux. Turbulence caused by radiative effects was better resolved in the 3-D cloud model than in the 2-D model. A change in domain size caused a shift in time of major con- vective events. For their radiation experiments, they differentiated between a no radiation, a local 1-D radiation and an averaged radiation application in a 2-D cloud model. Ice and mass flux, as well as the amount of condensed water, increased in the slab-averaged radiation application. Petch and Gray (2001) related this to destabilization due to the averaged cooling in areas where there should be no or less cooling in the local application, causing an increase in depth and the rate of development of the clouds. Overall, there were some differences between the averaged and the local radiation cases, but the larger one was found when comparing the radiation results to the no radiation simulation. Cole et al. (2005) embedded a 2-D cloud model in a general circulation model (GCM) with $4 \mathrm{~km}$ horizontal resolution. They used a two-stream approach for solar radiation and an emissivity approach for the longwave spectrum. They applied the 1-D radiation both as local and averaged applications and investigated the feedback to cloud development within a 6-month simulation. In addition, they compared the results to the standard GCM radiation scheme, which produces also an average radiation tendency per GCM grid box. They concluded that local application of radiative effects causes differences in the development of low and high clouds. The way the slab average radiation is applied (either calculated from the direct simulation of local effects or from the GCM itself) did not change cloud development in a significant way. The difference between the application of local and homogenized radiation was also addressed by Xiao et al. (2014), with a focus on stratocumulus to cumulus transition. Again, an increase in turbulence, due to the destabilization of the clouds by thermal radiation, was found. Xiao et al. (2014) state that because they only used a common 1-D approximation for the radiation calculation the effects might be larger with $3-\mathrm{D}$ radiation.

In addition to the thermal radiative impacts on cloud development on short timescales mentioned so far, studies by Muller and Held (2012) and Muller and Bony (2015) suggest that local thermal radiation is essential for triggering selfaggregation in radiative-convective equilibrium simulations. Emanuel et al. (2014) found that clear sky thermal cooling is a key component for self-organization.

Studies coupling thermal interactive 3 -D radiation to cloud resolving models are rare. Guan et al. (1997) investigated 3$\mathrm{D}$ thermal radiation effects in small cumulus clouds using a 2-D cloud model and the axially symmetric 3 -D radiative transfer model of Guan et al. (1995). Simulations with 3-D longwave radiative transfer were compared to a no radiation simulation. An increase in mean and maximum liquid water content was found. In addition, enhanced downward motion at the cloud sides and an increased upward motion in the cloud center developed in the $3-\mathrm{D}$ radiation case. At the end of their simulation, Guan et al. (1997) found an acceleration of the dissipation of the cloud with thermal radiation. Mechem et al. (2008) coupled the 3-D radiative transfer model SHDOM (Evans, 1998) to a 2-D cloud model. The ef- 
fects of 3-D thermal radiative transfer on stratocumulus and isolated shallow cumulus were studied. The tendencies of the difference between the 1-D and 3-D radiative transfer calculation were passed to the cloud model. They found an overall effect of thermal radiation on the development of the cloud field in comparison to a no radiation simulation, but the differences between 1-D and 3-D thermal radiation were small. Interactive radiation promoted deeper clouds, higher liquid water content, as well as cooler and dryer surface conditions. In the stratocumulus case, the main difference was a redistribution of the heating rates in the cloud field.

The microphysical aspect mentioned before will not be addressed in this study; however, for completeness, we will briefly point out what was found in the past. Harrington et al. (2000) and Marquis and Harrington (2005) showed that thermal emission enhanced cloud droplet growth by diffusion. An earlier onset of collision and coalescence of cloud droplets was found by Hartman and Harrington (2005a, b) when thermal radiation was considered. Recent studies of Brewster (2015) and de Lozar and Muessle (2016) emphasize the hypothesis that thermal radiation might influence droplet growth significantly and lead to a broadening of the droplet size spectra and thus enhance the formation of precipitation.

Most of the former studies of cloud-radiation interactions were based on 1-D radiation assumptions. The few studies using 3-D radiative transfer models are limited to using 2D cloud resolving models instead of full 3-D cloud models. This paper aims to address the interaction of radiation and clouds, including a comparison of the effects of 1-D and 3-D thermal radiation. For this purpose, 3-D interactive radiation (the Neighboring Column Approximation - NCA; Klinger and Mayer, 2016) was developed and integrated into the UCLA-LES (Stevens et al., 2005; Stevens, 2007) and a set of idealized simulations was developed, aiming to isolate the effect of 1-D and 3-D thermal radiation on clouds. The NCA is fast enough to allow really extensive 3-D thermal radiation studies for the first time.

In this paper, we extend former studies by applying a fully coupled 3-D radiative transfer method in a 3-D cloud model and compare 1-D and 3-D thermal radiative effects. Thermal radiative transfer is applied in a "local" and a "horizontally averaged" setup. We start with simulations of single clouds driven by a heat bubble disturbance and by comparing a no radiation simulation to $1-\mathrm{D}$ and 3-D local thermal radiation simulations, thus bridging the gap of the previous study of Guan et al. (1997) where only no radiation and 3$\mathrm{D}$ thermal radiation simulations were compared. In a second step, we extend our setup to a shallow cumulus cloud field at 50 and $100 \mathrm{~m}$ resolutions, thus increasing the resolution and domain size of previous studies and applying 3-D radiative transfer in a 3-D cloud model. The model and model setup are described in Sect. 2. The results are presented in Sect. 3.

\section{Simulation setup}

The University of California Los Angeles large eddy simulation model (UCLA-LES; Stevens et al., 2005; Stevens, 2007) is used for our analysis. The model has previously been successfully used to represent various typical cases, including BOMEX (Cheng et al., 2010), RICO (van Zanten et al., 2011) and DYCOMS (Stevens et al., 2005). The standard UCLALES includes bulk microphysics for warm clouds (Seifert and Beheng, 2001) and a 1-D radiation scheme (delta-fourstream approximation; Liou et al., 1988). The spectral integration is accounted for with a correlated-k molecular absorption parameterization ( $\mathrm{Fu}$ and Liou, 1992). In addition, the Monte Carlo spectral integration (MCSI; Pincus and Stevens, 2009) is used in this study for the simulation of the cumulus cloud field to save computational time. The UCLALES was adapted for 3-D local thermal radiation by implementing the NCA (Klinger and Mayer, 2016) for the calculation of 3-D thermal heating and cooling rates.

Two passive scalar tracers were implemented into UCLALES, following Park et al. (2016). With the help of the tracers, we performed an octant analysis (Park et al., 2016) to extract coherent structures in simulation data. For further analysis of the results, we used the cloud-tracking algorithm $\mathrm{Cb}$ TRAM (Zinner et al., 2008).

\subsection{UCLA-LES setup}

Two different types of idealized cloud studies have been performed, either with a single cloud or a complete nonprecipitating shallow cumulus cloud field.

\subsubsection{Single cloud}

A single cloud, induced by a heat bubble, is investigated to study the effects of thermal radiation on individual clouds. We compare the effects of a simulation without radiation ("No Radiation") to simulations with 1-D independent column approximation ("1-D Thermal ICA") and simulations with 3-D thermal radiation ("3-D Thermal NCA") using the NCA. For the simulation, the full thermal spectrum was simulated.

As the strength of the radiation effect on cloud development likely depends on the shape and dynamics of a cloud, we choose four different clouds for our investigation.

- The first is a weakly driven, axially symmetric cloud. The heat bubble is introduced by a elliptical-shaped volume of warmer air close to the surface. The temperature perturbation is $0.4 \mathrm{~K}$.

- The second is a weakly driven, non-symmetric cloud. The heat bubble is introduced by a uniform random perturbation varying between 0.0 and $0.8 \mathrm{~K}$ in the same elliptical-shaped volume as the weakly driven symmetric cloud, giving the same average perturbation of $0.4 \mathrm{~K}$ 
Table 1. Model setup for the single cloud simulations. CCN indicates cloud condensation nuclei.

\begin{tabular}{ll}
\hline Model variables & Value \\
\hline Number of grid boxes & $128 \times 128$ \\
Number of $z$ levels & 70 \\
Resolution & $50 \mathrm{~m}$ \\
Vertical stretching & $1 \%$ \\
Surface perturbation & $0.8 / 1.6 \mathrm{~K}$ \\
SST & $288 \mathrm{~K}$ \\
CCN & $70 \times 10^{6} 1 / \mathrm{kg}$ \\
Microphysics & warm, no rain \\
Variable output & every $100 \mathrm{~s}$ \\
Surface type & fixed SST \\
\hline
\end{tabular}

as above. The cloud is comparable in strength to the weakly driven, axially symmetric cloud.

- The third is a stronger-driven, axially symmetric cloud. The heat bubble is introduced by a elliptical-shaped volume of warmer air close to the surface. The temperature perturbation is $0.8 \mathrm{~K}$.

- The last is a stronger-driven, non-symmetric cloud. The heat bubble is introduced by a uniform, random perturbation varying between 0.0 and $1.6 \mathrm{~K}$ in the same elliptical-shaped volume as the stronger-driven symmetric cloud, giving the same average perturbation of $0.8 \mathrm{~K}$ as above. The cloud is comparable in strength to the stronger-driven, axially symmetric cloud.

A stable background profile was chosen in order to cause only moderate updraft velocities of a few meters per second. The simulation is performed over $80 \mathrm{~min}$. More setup details are summarized in Table 1. The random noise in the nonsymmetric cloud simulations was initialized with the same random seed in all simulations in order to simulate clouds of similar shape, which allows a direct comparison of the development of the clouds. The simulations are performed at $50 \mathrm{~m}$ horizontal resolution in a $6.4 \mathrm{~km}^{2} \times 6.4 \mathrm{~km}^{2}$ domain. A stretching of the vertical grid of $1 \%$ was applied starting at $10 \mathrm{~m}$ height.

\subsubsection{Shallow cumulus cloud field}

Large-scale simulations of a shallow cumulus cloud field in a $50 \mathrm{~km} \times 50 \mathrm{~km}$ domain with 50 and $100 \mathrm{~m}$ horizontal resolutions have been performed. The environment was that of a warm ocean surface. All simulations are run for $30 \mathrm{~h}$ at Deutsches Klima Rechenzentrum (DKRZ) in Hamburg on the Mistral supercomputer (Intel Haswell) on 512 cores. We focused on the effects of thermal radiative heating and cooling on the clouds themselves. Therefore, surface fluxes of latent $\left(180 \mathrm{~W} \mathrm{~m}^{-2}\right)$ and sensible heat $\left(18 \mathrm{~W} \mathrm{~m}^{-2}\right)$ were fixed throughout the simulation. The initial atmospheric profiles
Table 2. Model input for shallow cumulus cloud field simulations.

\begin{tabular}{ll}
\hline Model variables & Value \\
\hline Number of grid boxes & $1024 \times 1024 / 512 \times 512$ \\
Number of $z$ levels & 90 \\
Resolution $x, y$ & $50 / 100 \mathrm{~m}$ \\
Resolution $z$ & $30 \mathrm{~m}$ \\
Vertical stretching & $1 \%$ \\
CCN & $150 \times 10^{6} 1 / \mathrm{kg}$ \\
Microphysics & warm, no rain \\
Variable output & every $300 \mathrm{~s}$ \\
Latent heat & prescribed: $180 \mathrm{~W} \mathrm{~m}^{-2}$ \\
Sensible heat & prescribed: $18 \mathrm{~W} \mathrm{~m}^{-2}$ \\
Restart & $10800 \mathrm{~s}$ \\
\hline
\end{tabular}

for these simulations were taken from Stevens (2007). A stretching of the vertical grid of $1 \%$ was applied, starting at $100 \mathrm{~m}$ height. We allow for warm microphysics (Seifert and Beheng, 2001) but omit the development of rain to prevent possible feedbacks that rain might cause (e.g., cold pool dynamics) and concentrate rather on radiative effects. Due to the high computational costs of radiation simulations, we used the Monte Carlo Spectral Integration (MCSI, Pincus and Stevens, 2009) in a version adapted for 3-D local radiation described in Jakub and Mayer (2016). Further details are given in Table 2 .

Again, we compare different radiation types (1-D Thermal ICA and 3-D Thermal NCA). Those are the "local radiation" applications, where heating and cooling act locally where they are generated. In addition, we "averaged" the thermal heating and cooling of the 1-D Thermal ICA and 3-D Thermal NCA radiation solution in each time step in each layer ("1-D Thermal AVG" and "3-D Thermal AVG"). These averaged heating rates are then applied in the entire layer to clear sky and cloudy regions. This allows us to separate the effects of local heating/cooling from the systematically larger cooling that is introduced by thermal radiation. Additionally, we apply a "constant cooling" of $2.6 \mathrm{~K} \mathrm{day}^{-1}$ throughout the simulation in the modeling domain. The magnitude of the cooling was chosen specifically to be comparable to the cooling introduced by the local radiation simulations. The constant cooling differs from the "averaged radiation" simulations in the profile of the cooling. The averaged radiation simulations cause more cooling in the cloudy layers.

All simulations are restarted and analyzed after a $3 \mathrm{~h}$ initialization run. Until $3 \mathrm{~h}$, the initial simulation is driven by 1-D solar and thermal radiation. From the restart time on, we switch on one of the five thermal radiation applications or switch radiation off, thus skipping the spinup. At $3 \mathrm{~h}$, the first clouds form in the initial run. 

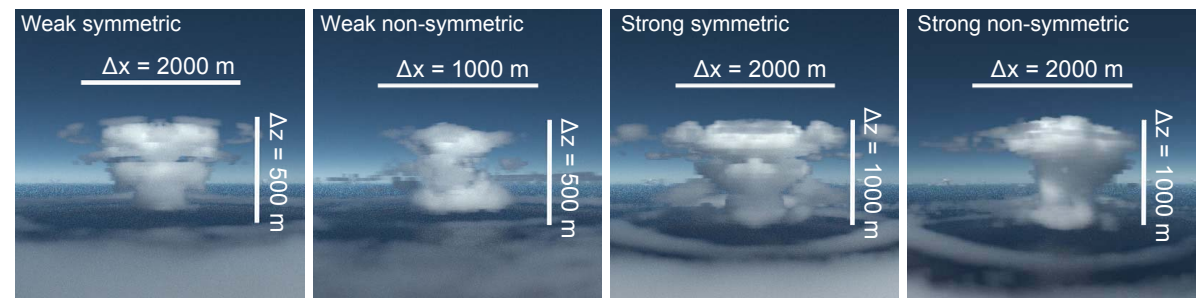

Figure 2. Visualization of the four single cloud simulations (first: weak symmetric; second: weak non-symmetric; third: strong symmetric; fourth: strong non-symmetric). The snapshot of the cloud field is taken at $40 \mathrm{~min}$ of the simulation of the 3-D Thermal NCA case. The visualization was performed with the 3-D radiative transfer model MYSTIC (Mayer, 2009). The clouds in the background are a feature of the visualization and do not occur in the LES simulation of the single clouds. Clouds close to the surface are neglected in our analysis. Please note that the clouds are rather oblate, although they appear stretched due to the perspective used in this visualization.

\subsection{Cb-TRAM cloud-tracking algorithm}

To quantify some statistics on the cloud size, lifetime and number of clouds in the simulations, we use a cloud-tracking algorithm to track individual clouds over time. Cb-TRAM was originally set up to work with satellite imagery by Zinner et al. (2008) but is easily adapted to any other 2-D information. Here, fields of the liquid water path are tracked. $\mathrm{Cb}$-TRAM identifies objects as contiguous areas with a specific common characteristic. We set two thresholds to define a cloud: first, only cloud columns of a liquid water path larger than $20 \mathrm{~g} \mathrm{~m}^{-2}$ are considered; second, a cloud must consist at least of 16 grid-connected boxes. Objects defined this way at one time step are identified in the water path field of the next time step using an optical flow analysis of the liquid water field deformation and a simple object overlap analysis. This way, cloud objects are detected and tracked over time, allowing us to estimate cloud size and lifetime distributions.

\section{Simulation results}

\subsection{Single cloud simulations}

Figure 2 provides a first impression of the four different single cloud simulations. The visualization shows the weak symmetric, weak non-symmetric, strong symmetric and strong non-symmetric single cloud simulations at $40 \mathrm{~min}$ for the 3-D Thermal NCA simulation. The following section provides a detailed analysis of the development of these single clouds for different radiation setups.

A comparison of the time development of liquid water, vertical velocity and cooling rates is shown in Figs. 3 and 4.

The liquid water path and cooling rates are sampled for the initial cloud only ("conditionally sampled"). During the simulations, new clouds form close to the surface (see Fig. 2, which we ignore in our analysis). A running average over $300 \mathrm{~s}$ was applied to the time series in order to smooth the results. A gray shaded area covers the first $40 \mathrm{~min}$ of the simulations in Figs. 3 and 4. During this time, the cloud development is dominated by the heat perturbation of the warm bub-
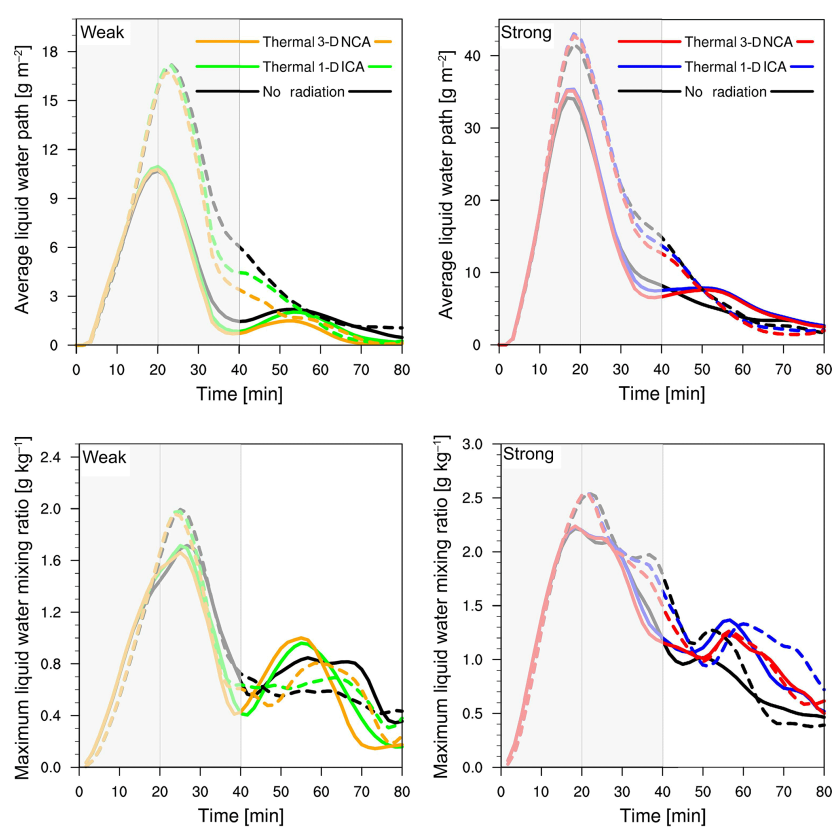

Figure 3. Time development of the conditionally sampled liquid water path and maximum liquid water mixing ratio for the simulations of the single clouds. Only liquid water belonging to the single cloud was considered. The left column shows the weaker forced single clouds; the right column shows the stronger forced single clouds. Solid lines represent the non-symmetric cloud; dashed lines represent the symmetric cloud.

ble. Updraft vertical velocities are strong in this initial stage. We only expect a significant effect of thermal radiation on cloud development after that initial stage. Summing up the thermal cooling in our simulations over time, we found that $40 \mathrm{~min}$ is about the time it takes for the thermal cooling to compensate the original heat perturbation of the bubble. This time period is roughly the same in the strong and weakly forced cases, because the stronger forced single clouds contain more liquid water and therefore more thermal cooling.

Both the symmetric and non-symmetric clouds show the impact of thermal radiation on the cloud development. Fo- 

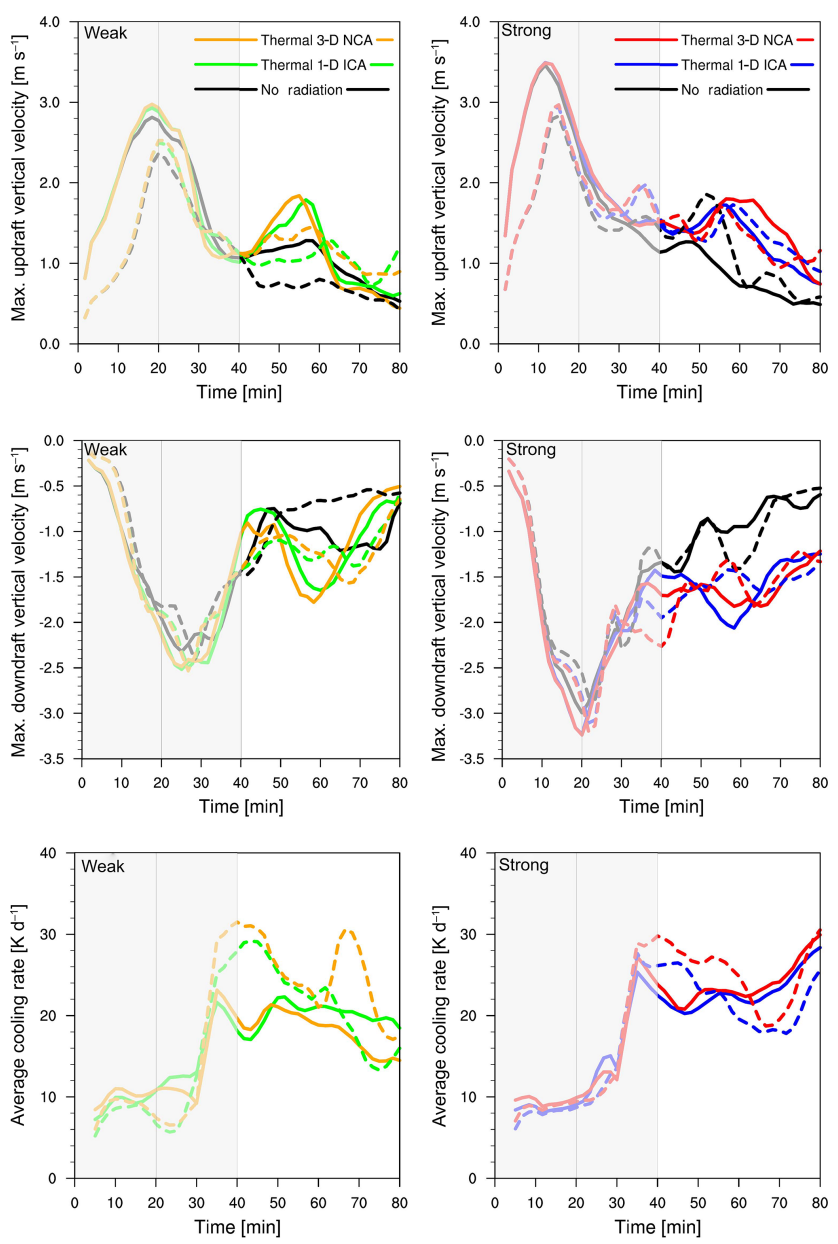

Figure 4. Time development of maximum updraft and downdraft vertical velocity, as well as conditionally sampled cooling rates of the single cloud simulations for the simulations of the single clouds. Cooling rates were sample at the single cloud only. The left column shows the weaker forced single clouds; the right column shows the stronger forced single clouds. Solid lines represent the nonsymmetric cloud; dashed lines represent the symmetric cloud.

cusing on the liquid water path (top row of Fig. 3) we can split the cloud development into three stages. Both cloud types show a fast development at the beginning (up to about $20 \mathrm{~min}$ in the gray shaded area until first gray line). We refer to this first development of the cloud as the "first stage". During that time, the development is dominated by the heat perturbation at the surface. Both liquid water quantities develop similarly in all simulations, with only few differences due to thermal radiation.

After the first stage, the liquid water path decreases and, finally, from about 30 min onward the cloud stays rather constant at a certain height and does not rise any further. Updrafts become weaker (top row of Fig. 4) and radiation acts more significantly on the cloud. All simulations show that the liquid water path (top row of Fig. 3) is reduced in this "second stage" (from about 20 to $40 \mathrm{~min}$ ). The "last stage"

of cloud development (about 40 to $80 \mathrm{~min}$ ) is dominated by a second growth period of the cloud. In this stage, thermal radiation can act on the cloud. Both non-symmetric clouds show a second rise in the liquid water path in the case of the stronger forced cloud exceeding the No Radiation simulation. When comparing the development of the liquid water path to the development of maximum liquid water mixing ratio over time (lower row of Fig. 3), the rise in liquid water mixing ratio in the last phase becomes more evident. Maximum values of liquid water mixing ratio in the radiation simulations exceed the No Radiation simulation in this last phase. Looking at the location of these maximum values of liquid water mixing ratio and the shape of the cloud (not shown), we find that clouds become narrower (have a reduced horizontal extent) over time when radiative effects are accounted for and maxima of liquid water mixing ratio are enhanced in the center of the cloud. Vertical velocities show stronger upward and downward values for both thermal radiation simulations. The upward and downward motions in the first stage results from the initial temperature perturbation and the resulting overturning circulation.

Looking at the differences between 1-D Thermal ICA and 3-D Thermal NCA, we find different amounts of cooling in the 3-D Thermal NCA radiation simulation which affects the further development of the cloud (bottom row of Fig. 4). Differences occur in terms of liquid water when comparing both thermal radiation simulations. The differences are small but in general slightly stronger in the case of the 3-D Thermal NCA simulation. In the last stage, differences in vertical velocity between the No Radiation and the thermal radiation simulations are evident. Both radiation simulations show stronger upward and downward vertical velocities. Vertical velocities are usually a bit stronger in the case of 3-D Thermal NCA radiation than in the case of 1-D thermal radiation. Combining the development of liquid water with upward and downward vertical velocities (top and middle row of Fig. 4), the data suggest that a change in cloud circulation is induced from the second stage onward by the effects of thermal radiation, which enhances updraft vertical velocities in the cloud cores, thus strengthening the cloud development and, at the same time, inducing stronger downdraft vertical velocities at the cloud sides.

The region of subsiding motion around a cloud, the "subsiding shell" is known from previous work of Heus and Jonker (2008), Jiang et al. (2006) and Small et al. (2009). Heus and Jonker (2008) studied the subsiding shell of cumulus clouds from measurement data in comparison to model simulations and concluded that negative buoyancy at the cloud sides causes the subsiding shell to develop. Jiang et al. (2006) and Small et al. (2009) compared measurement data and simulations of small cumulus clouds to investigate the effect of different aerosol concentrations on cloud lifetime. They found a stronger subsiding shell due to enhanced evaporative cooling. Stronger downward motion at cloud sides due to thermal radiation was found by Guan et al. (1997). 

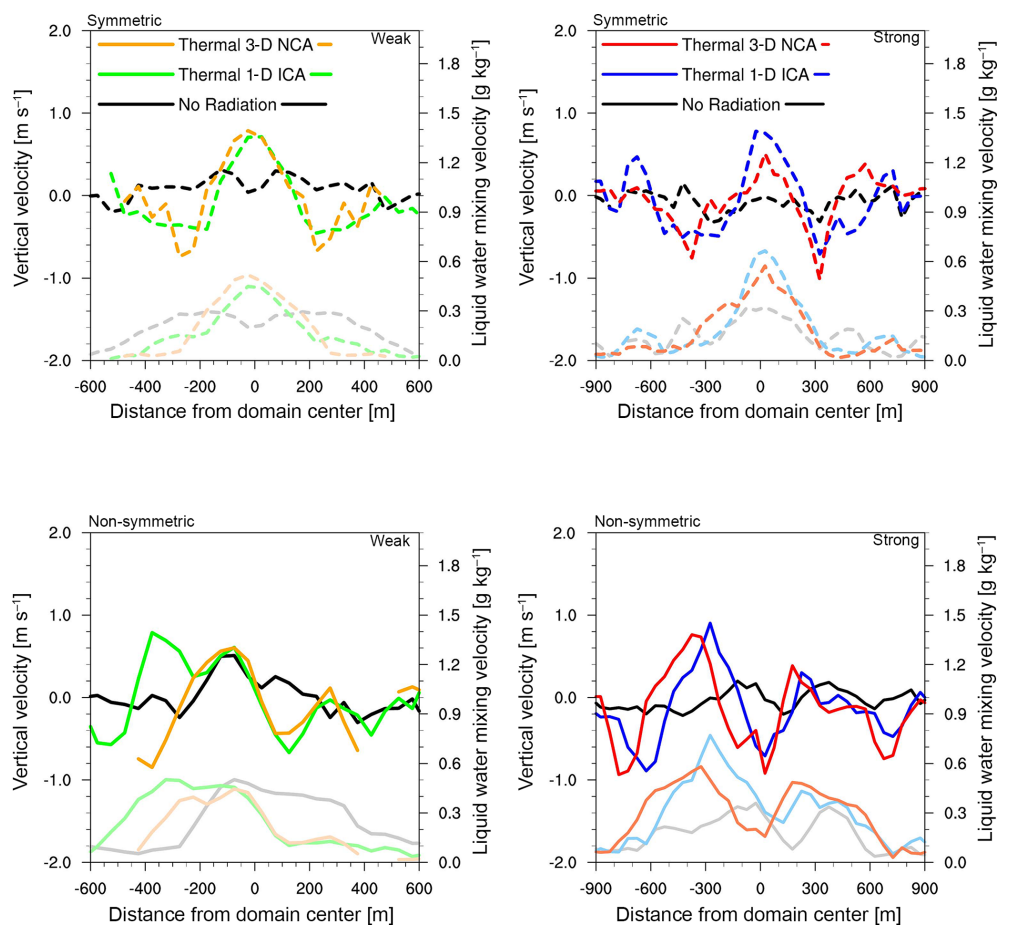

Figure 5. Height- and time-averaged transects of liquid water mixing ratio and vertical velocity. Liquid water mixing ratio is shown in pale colors on the bottom; the vertical velocity is shown in middle of each figure. The corresponding axes are on the right and left, respectively. The time averaging was performed over $3 \mathrm{~min}$ at around $60 \mathrm{~min}$ simulation time. The vertical average was taken in the middle of the vertical extent of the cloud to cover cloud side areas. Dashed lines show the symmetric cloud; solid lines show the non-symmetric cloud.

To further investigate this question and the development of the cloud, with a special focus on the possible change in cloud circulation and the subsiding shell, Fig. 5 shows transects of liquid water (bottom of each figure) and vertical velocity (center of each figure) in the last stage of cloud development. Both quantities displayed are averages of the 900$1200 \mathrm{~m}$ height layer. In the time series analysis, the cloud development is accompanied by stronger updrafts and stronger downdrafts in the simulations with radiation. Figure 5 shows the stronger upward and downward motions in the transect. The subsiding shells are clearly visible at the cloud side region. Vertical velocities increase from $-0.1 \mathrm{~m} \mathrm{~s}^{-1}$ in the No Radiation simulation to about -0.8 to $-1.0 \mathrm{~m} \mathrm{~s}^{-1}$ in the simulations with radiation, peaking for the 3-D thermal radiation simulation, although the difference between 1-D and 3-D radiation is modest. Liquid water content is enhanced in the simulations with radiation in the cloud center, and the cloud is narrower (Fig. 5). This is in agreement with the results of the time development of the liquid water path and maximum liquid water (Fig. 3) that indicated narrower clouds with enhanced liquid water content in the cloud center.

Finally, Fig. 6 shows the horizontally averaged vertical profile of negative and positive buoyancy, sampled in the cloudy region (all grid boxes where liquid water is larger than zero). Data are sampled for $8 \mathrm{~min}$, starting after $50 \mathrm{~min}$ of the simulation. All simulations including thermal radiation show
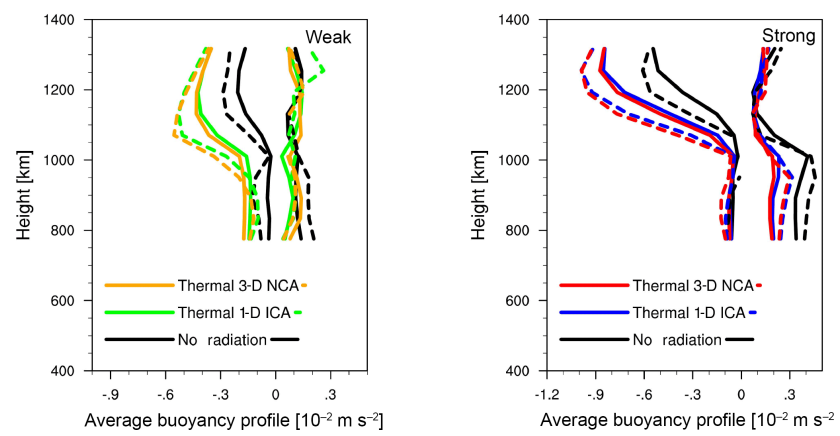

Figure 6. Positive and negative buoyancy profile sampled at 5058 min of the simulation in the cloudy area. Dashed lines show the symmetric cloud; solid lines show the non-symmetric cloud.

stronger negative buoyancy which is slightly larger in the 3D Thermal NCA case than in the 1-D case. This negative buoyancy is due to the thermal cooling at cloud tops and, in the case of 3-D Thermal NCA radiation, at cloud sides. The negative buoyancy can cause the observed subsiding motion, as already found by Heus and Jonker (2008) and Small et al. (2009). In addition, the stronger horizontal buoyancy gradient (difference between positive and negative buoyancy in Fig. 6) generates enhanced turbulence and lateral mixing and therefore stronger evaporation. The stronger evaporation ex- 
plains the narrowing of the clouds in the horizontal direction seen in Fig. 5. Enhanced evaporation can cause an additional cooling and therefore a positive feedback to the already existing horizontal buoyancy gradient. Whether thermal radiation itself or the enhanced evaporation contributes more to the formation of the subsiding shell cannot be said from our current simulations. However, it is certain that thermal radiation strengthens the development by generating negative buoyancy.

These results confirm what was found by Guan et al. (1997). They also found an increase in liquid water, both in terms of average and maximum values and stronger downward motion at the cloud side. While Guan et al. (1997) compared a no radiation simulation to a 3-D thermal radiation simulation of a symmetric cloud, we include 1-D radiative transfer in our study and extend the study to non-symmetric clouds. The differences between 1-D and 3-D thermal radiative transfer are small. However, 3-D thermal radiation causes a slightly stronger effect than 1-D thermal radiative transfer. As the effect of 3-D thermal radiation is the additional cloud side cooling, the magnitude of this cooling determines how strong the 3-D thermal radiation effects are. The amount of cooling in turn is related to the cloud side area. As Fig. 2 shows, our clouds are rather oblate, which reduces the 3-D radiation effect since the side area is small compared to the top area. In addition, the limitations of the NCA, as discussed later in this paper (Sect. 3.2.4), might cause some neglect of the cloud side cooling. We summarize therefore that we can confirm previous findings that thermal radiation changes the cloud circulation and enhances liquid water content with 1-D thermal radiation being nearly as efficient as 3$\mathrm{D}$ thermal radiation. The magnitude of the 3-D effect might depend significantly on the shape of the cloud (cloud type) and requires further study.

\subsection{Shallow cumulus cloud field model experiments}

In this section, we explore the effects of thermal radiation on the development and organization of shallow cumulus clouds in the $50 \mathrm{~km} \times 50 \mathrm{~km}$ domain at $50 \mathrm{~m}$ horizontal resolution. Figure 7 shows the cloud field for three of the six performed simulations. The figure shows a time snapshot at $20 \mathrm{~h}$ of the simulations. We can already see in this snapshot that clouds organize differently depending on the radiation application used.

Figure 8 shows the temporal development of cloud fraction and maximum liquid water mixing ratio from the restart time until $30 \mathrm{~h}$.

In addition to the local 3-D and 1-D thermal radiation cases, the averaged and fixed (constant cooling) radiation scenarios are shown. In the No Radiation simulations, cloud cover stays constant at about $10-12 \%$ from $8 \mathrm{~h}$ on with a slight decrease towards the end of the simulation. Maximum liquid water mixing ratio is less in the No Radiation simulation, compared to the simulations with radiation $\left(1.9 \mathrm{~g} \mathrm{~kg}^{-1}\right.$

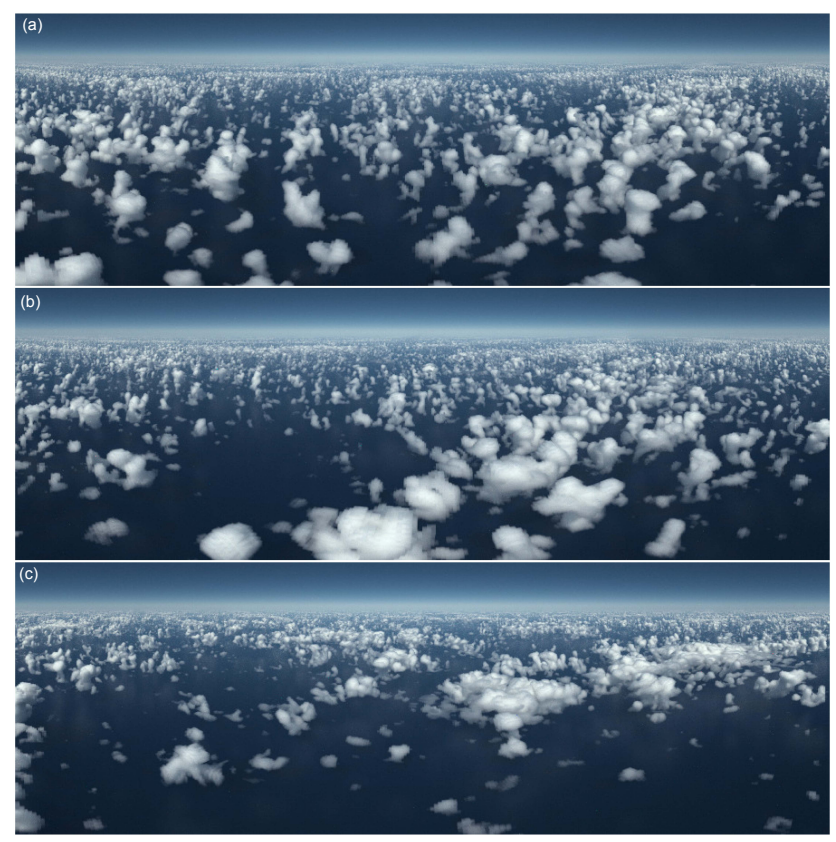

Figure 7. Visualization of the cumulus cloud field of the constant cooling (a), 3-D Thermal AVG (b) and 3-D Thermal NCA (c) simulations. The snapshot of the cloud field is taken at $20 \mathrm{~h}$ of the simulation. The visualization was performed with the 3 -D radiative transfer model MYSTIC (Mayer, 2009).

vs. $3.6 \mathrm{~g} \mathrm{~kg}^{-1}$ ). Differences are also found between the local radiation simulations and the averaged radiation simulations. Cloud cover increases more rapidly in the averaged radiation simulations compared to the local ones. The maximum of liquid water mixing ratio, however, shows an opposite development. It is therefore likely that clouds organize differently, depending on the treatment of radiation. The two gray lines at 10 and $20 \mathrm{~h}$ indicate stages where the development of the simulation changes. While all simulations with radiation perform nearly the same until $10 \mathrm{~h}$, they start to differentiate afterwards. Maximum liquid water mixing ratio exceeds $2 \mathrm{~g} \mathrm{~kg}^{-1}$ after $20 \mathrm{~h}$. In a rain-permitting simulation, rain would likely form at that time. We ran the simulations for $30 \mathrm{~h}$ to see what would theoretically happen to the clouds, but as we are aware that the simulations become more and more unrealistic from $20 \mathrm{~h}$ on, our main analysis will be on the time interval between restart and $20 \mathrm{~h}$. After $20 \mathrm{~h}$, a strong increase in cloud fraction in all simulations with radiation is found. This increase is particularly strong for the two averaged radiation simulations. Liquid water mixing ratio (and the liquid water path, not shown) starts varying more rapidly, indicating stronger formation of clouds and their decay.

Figure 9 shows the temporal development of the liquid water path (sampled in the entire domain), maximum vertical velocity and cloud base and top height until $20 \mathrm{~h}$. The liquid water path increases with time in all simulations. The increase is strongest in the case of 3-D Thermal NCA. Each of 

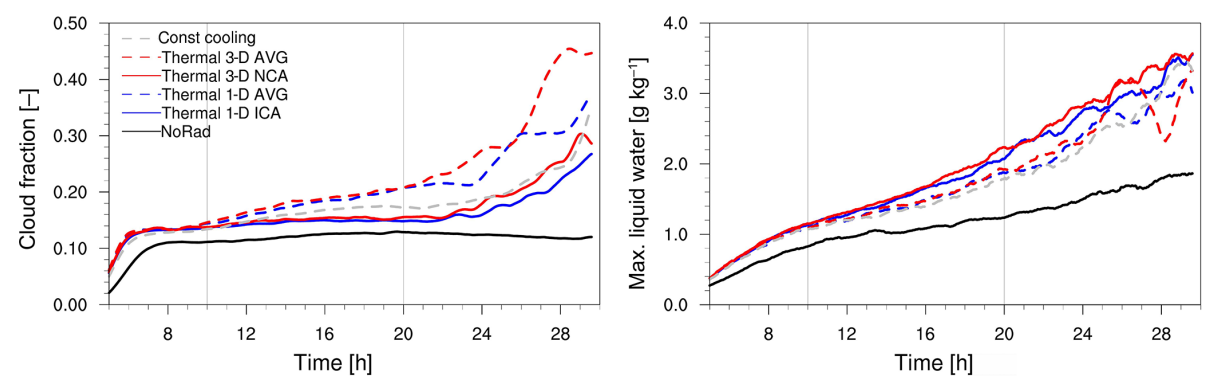

Figure 8. Time development of cloud fraction and maximum liquid water mixing ratio from 5 to $30 \mathrm{~h}$. The two gray lines (at 10 and $20 \mathrm{~h}$ ) separate the development in different periods for further analysis.
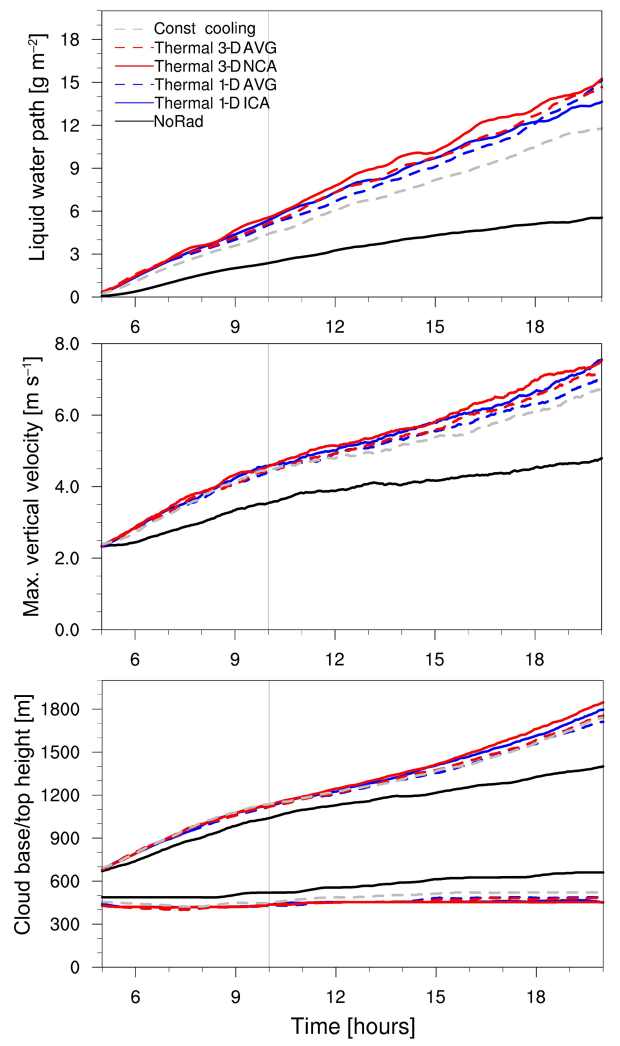

Figure 9. Time development of the liquid water path, maximum vertical velocity and cloud base and cloud top height. The gray line (at $10 \mathrm{~h}$ ) separates the development in different periods for further analysis.

the local thermal radiation simulations produces more liquid water than its averaged counterpart. However, these differences are small. The different development of cloud cover and liquid water in the averaged and local thermal radiation simulations results in smaller differences between these two setups. The liquid water path increases less in the simulation with constant cooling. The maximum vertical velocities are weaker in the No Radiation simulation and cloud vertical extent is smaller with a heightened cloud base and lower cloud tops. All quantities increase over time. Due to the missing

cooling, less water condenses; thus, the number of clouds, cloud cover and a liquid water path are reduced. The higher cloud base is also a result of the missing cooling which leads to a warmer temperature profile in the No Radiation simulation. The smaller increase in cloud top height might be linked to reduced updraft velocities and missing destabilization (due to thermal cooling) in the No Radiation simulation (see Sect. 3.2.1).

To study whether the observed differences are simply caused by the systematic change introduced by 1-D or 3-D radiation, or if the local effects are relevant, we compare the averaged and local thermal radiation simulations. Although the averaged amount of cooling per domain is (in the beginning of the simulation) the same for both 1-D Thermal ICA and 1-D Thermal AVG (or 3-D Thermal NCA and 3-D Thermal AVG) simulations, the averaged radiation simulations produce higher cloud cover than their corresponding 1-D or 3-D local radiation simulation. The liquid water path and maximum liquid water content develop in the opposite direction: both are lower for the averaged radiation simulations until $20 \mathrm{~h}$. The development of all quantities shown here can be related to the location where thermal radiation acts. In the local radiation simulations, cooling (and some warming at the cloud bottom) acts directly at the cloud edges. Cooling rates can locally be up to several hundred Kelvin per day and can destabilize the cloud layer, thus promoting updrafts, more condensation and an increase in cloud height. For the averaged radiation simulations, cooling occurs in the cloud layers, but as it is averaged and applied everywhere in a layer, independent of the location of the clouds, a destabilization occurs in the whole area. However, the destabilization of the averaged radiation simulations is weaker in the clouds than the local destabilization of the local radiation simulations. Finally, in the constant cooling simulation, the cooling is distributed equally over all heights. Cooling in the cloud layer is thus smaller compared to the simulations with local or averaged radiation, which explains the lower liquid water path.

\subsubsection{Boundary layer and cloud layer development}

We investigate the development of the boundary layer and cloud layer by examining the profiles of different quantities 

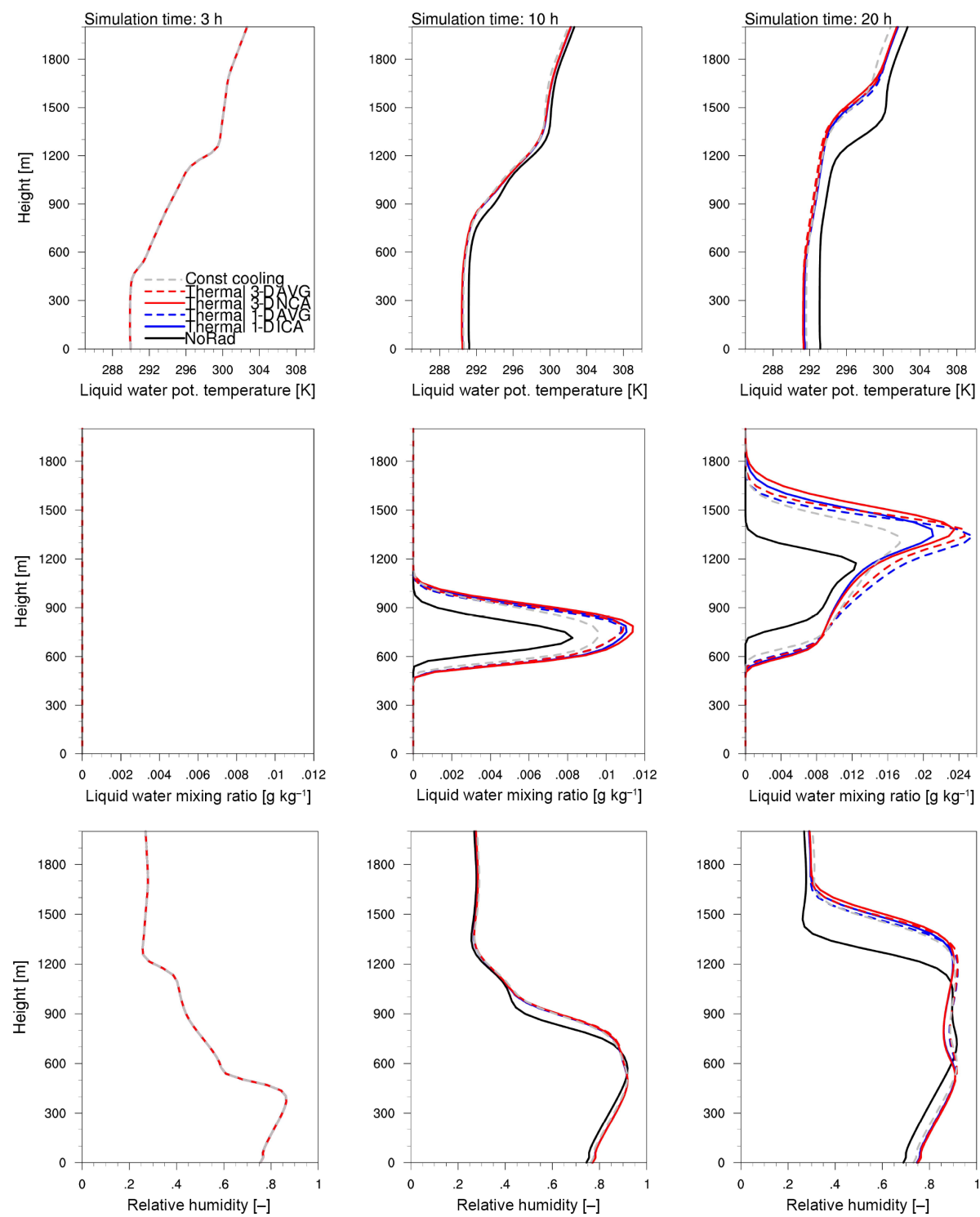

Figure 10. Time-averaged profiles of liquid water potential temperature, liquid water mixing ratio and relative humidity. The profiles are shown at the restart time $(3 \mathrm{~h})$ as a $3 \mathrm{~h}$ average centered at 10 and $20 \mathrm{~h}$ of the simulations.

at three time periods of the simulation. Starting with the initial profile at the restart time $(3 \mathrm{~h})$, we show in addition the averaged profile from 9 to $11 \mathrm{~h}$ (noted as $10 \mathrm{~h}$ ) and from 19 to $21 \mathrm{~h}$ (noted as $20 \mathrm{~h}$ ).

The initial profiles at $3 \mathrm{~h}$ (Figs. 10 and 11, first column) show the typical profiles of a boundary layer over a warm ocean surface. No clouds have developed yet at this stage. The profile of liquid water potential temperature shows a well-mixed layer (up to $400 \mathrm{~m}$ ), the conditionally unstable layer (up to $1200 \mathrm{~m}$ ), as well as the inversion layer at about $1100 \mathrm{~m}$ height. Relative humidity increases with height at first, before decreasing from $400 \mathrm{~m}$ height to the inversion, indicating the entrainment of dry air from above the inversion. Typical for the boundary layer over a warm ocean, tur- bulence is produced by buoyancy in the layer close to the warm ocean (rising thermals). The upward motion of this low layer can also be seen in the updraft velocity in the lower layer until $400 \mathrm{~m}$ height (Fig. 11, middle panel).

The first clouds appear shortly after the restart in all simulations. From this time on, thermal radiation (that is, cloud top cooling and cloud bottom warming and, in the case of 3-D Thermal NCA, cloud side cooling) changes the development of the boundary layer and of the clouds themselves. Due to the imposed constant surface fluxes of latent and sensible heat, the atmosphere warms over time. When thermal radiation is applied in the simulations, this warming is partially compensated by thermal cooling. At $10 \mathrm{~h}$, the whole atmosphere is about $1 \mathrm{~K}$ cooler in the thermal radiation simula- 

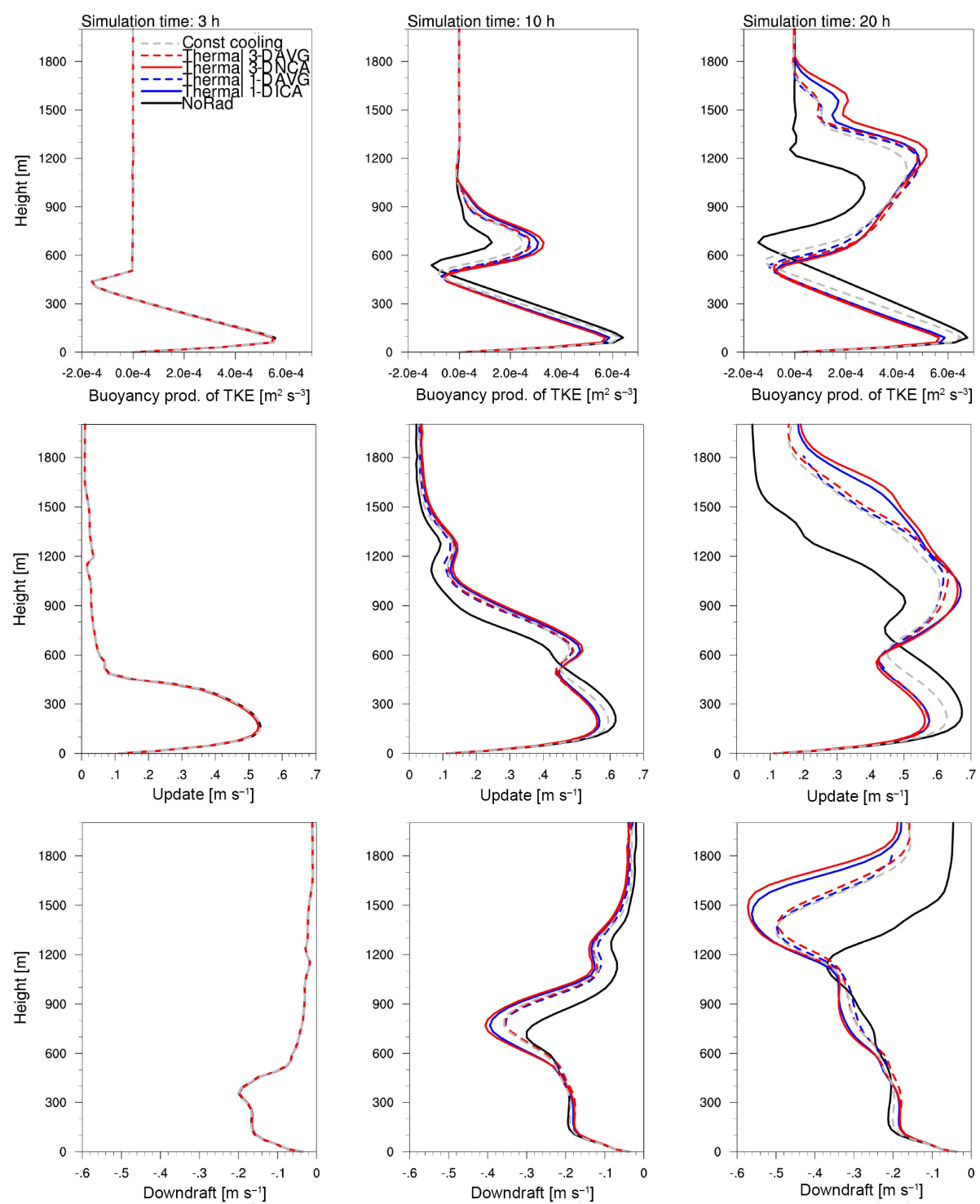

Figure 11. Time-averaged profiles of buoyancy production of the turbulent kinetic energy (TKE) and updraft and downdraft vertical velocity.The profiles are shown at the restart time $(3 \mathrm{~h})$ as a $3 \mathrm{~h}$ average centered at 10 and $20 \mathrm{~h}$ of the simulations. Updraft and downdraft vertical velocities were extracted from the 3-D data following Park et al. (2016).

tions (see Fig. 10) which in turn leads to a higher relative humidity and more condensation of water vapor. The increase in liquid water over time was already shown in Fig. 9. Here, in addition to the increased liquid water (which is stronger for the 3-D Thermal NCA radiation case at $10 \mathrm{~h}$ although differences are small), a deepening of the cloud layer occurs in the simulations including thermal radiation. Thermal cooling at the cloud boundary (local radiation simulations) and in the cloud layer (averaged radiation simulations) causes more condensation. The constant cooling simulations produces less water, because the cooling is not directly produced by the clouds but imposed in the simulation setup in the whole atmosphere.
The cooling at cloud tops (and cloud sides in the 3-D radiation case) as well as the bottom warming leads to a destabilization of the cloud layer, promoting the development of clouds by increased buoyancy (Fig. 11, second column). Turbulence that is initially only produced through surface-fluxinduced buoyancy tendencies is now additionally produced in the cloud layer. Both local radiation simulations show more buoyancy production than the averaged radiation simulations, again with the 3-D Thermal NCA simulation showing slightly stronger values than the 1-D Thermal ICA simulation. Due to the increased buoyancy in the simulations with radiation, upward velocities in the clouds are stronger (second column of Fig. 11, middle panel). Furthermore, all simulations with radiation produce stronger downdraft vertical 
velocities in the subsiding shells, especially the local radiation simulations (Fig. 11, bottom panel).

The difference in the temperature profiles between the No Radiation and the simulations with radiation increases (up to $3 \mathrm{~K}$ ), which again leads to an increase in relative humidity in the subcloud layer and more condensation. We note here that, in the cloud layer, the relative humidity decreases in the local radiation simulations, because the liquid water mixing ratio is higher, although the temperature is lower. The production of the turbulent kinetic energy (TKE) through buoyancy is shifted upward into the cloud layer and upward velocities increase in the cloud layer, which becomes deeper (see the deepening of liquid water profile; Fig. 10). While at the beginning of the simulation, the 3-D Thermal NCA simulations produced the largest amount of liquid water, the averaged radiation simulations produce the largest amount at the end of the simulation. The development of liquid water, relative humidity and the TKE production by buoyancy and the development of vertical velocities suggest that more mixing/entrainment of dry air from above the cloud layer occurs in the local radiation simulations.

We summarize therefore that 1-D Thermal and 3-D Thermal heating and cooling in clouds destabilize the cloud layer, promoting the development of strong updraft cores and the transport of water vapor into the cloud layer. In addition, the thermal cooling of the atmosphere leads to enhanced condensation. Mixing in the cloud layer is stronger. In addition to the stronger updraft velocities, downdrafts increase as well.

The local cooling at the cloud boundary itself in the local radiation simulations (in comparison to the averaged radiation effect) increases the earlier-described development by destabilizing the cloud layer locally in the clouds more than in the averaged radiation simulations. Entrainment is stronger in the local radiation simulations, causing less condensation and lower relative humidity. The simulation with constant cooling usually shows the weakest effect of all simulations with radiation.

We hypothesize that thermal radiation, and especially the localized thermal heating and cooling (as was already shown for the single cloud simulation), leads to stronger development of the cloud circulation in terms of updrafts and subsiding shells. 3-D Thermal NCA radiation, in comparison to 1-D thermal radiation, shows a slightly stronger increase of these shown effects by an additional cloud side cooling and overall stronger cooling in the modeling domain.

\subsubsection{Cloud development}

The preceding section (Sect. 3.2.1) analyzed the effects of thermal radiation on the development of the cloud-topped boundary layer. In this section, we further investigate the effects of thermal radiation on cloud development. It was shown before that the cloud circulation changes due to the effects of thermal radiation, promoting updrafts and subsiding shells, a deepening of the clouds and, depending on the radiation type, increased liquid water within the clouds. Another hypothesis raised earlier is the possible organization of clouds (see the beginning of Sect. 3.2) due to thermal radiation. In addition, thermal radiation may alter cloud lifetime. In the following, we will address these possible changes.

\section{Cloud circulation}

Results from the single cloud simulation and the statistical analysis of the cloud field simulations suggest that a change in the cloud circulation occurs, promoted by thermal radiative heating and cooling at the clouds. Stronger updrafts and stronger downdrafts/subsiding shells are expected due to the destabilization of the cloud layer and by thermal cooling of the clouds. Therefore, changes in the cloud circulation are expected to be stronger for the simulations with local 1-D Thermal ICA and 3-D Thermal NCA radiation, compared to the horizontally averaged radiation simulations. All simulations with radiation are expected to show stronger circulation features than the No Radiation simulation.

For the cloud field simulations, we used the octant analysis described by Park et al. (2016) to extract updrafts and subsiding shells from our simulations. By the signs of flux perturbations, eight parts (octants) are derived from the spatial field of three variables (vertical velocity and two passive scalars). Those octants include updrafts and subsiding shells/downdrafts (note that the analysis does not separate downdrafts inside clouds and subsiding shells). The analysis is restricted to cloudy layers (layers where at least one grid box has a liquid water mixing ratio larger than $0.1 \mathrm{~g} \mathrm{~kg}^{-1}$ ). Figure 12 shows the averaged and maximum updraft and downdraft velocities over time. Updrafts are stronger in all thermal radiation simulations compared to the No Radiation case. The updraft velocities of the local radiation simulation are slightly stronger than the updrafts in the averaged radiation simulations. The local radiation simulations produce stronger subsiding shells, noted in the averaged as well as maximum values. Updrafts and downdrafts in the 3-D radiation cases are in general slightly stronger than their 1-D counterparts. Therefore, an overall stronger circulation, induced by local heating and cooling, is found. These results agree with the increase in buoyancy production, the development of relative humidity and upward vertical velocity as shown in Sect. 3.2.1. In terms of updrafts and subsiding shells, we find the constant cooling simulations produces similar results to the averaged radiation simulations.

\section{Cloud organization}

Apart from the changes in the cloud circulation, clouds organize differently. Cloud cover and liquid water developed differently for the individual simulations. The horizontally averaged radiation simulations showed larger cloud cover over time than the local radiation simulations. 

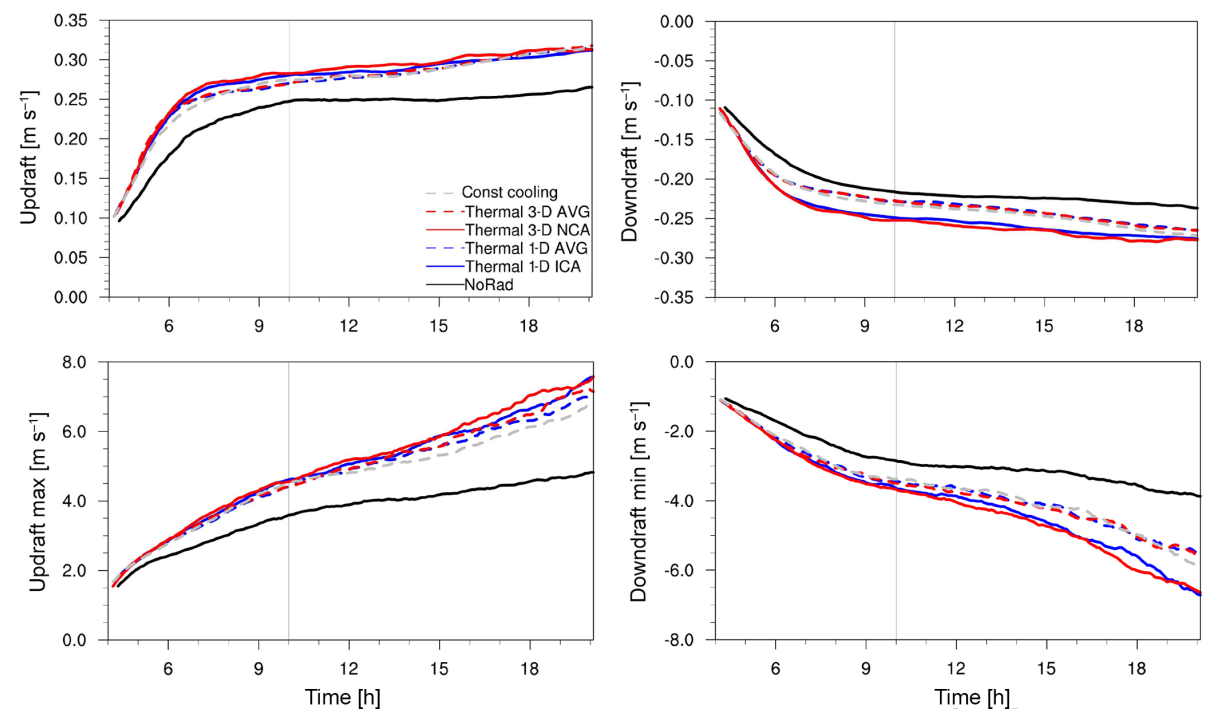

Figure 12. Time series of averaged vertical velocity as well as maximum updraft and downdraft vertical velocity of updrafts and downdrafts.

We investigate the cloud fields at 15 and $20 \mathrm{~h}$ of the simulations (Fig. 13). Comparing the No Radiation simulation to the thermal radiation simulation we see smaller, less deep and fewer clouds. As seen before, the simulations with constant cooling or averaged radiation show a similar behavior. Deeper clouds with higher liquid water content and a higher number of clouds are found here. At $20 \mathrm{~h}$ there seems to be a tendency for patches to form. The local radiation simulations show a completely different development. At $15 \mathrm{~h}$, we can see a separation into cloud-free and cloudy areas. The clouds are larger than in the averaged radiation simulations. This development continues and leads to cell structures at $20 \mathrm{~h}$, similar to those usually found due to cold pool dynamics (e.g., Sharon et al., 2006; Xue et al., 2008; Savic-Jovcic and Stevens, 2008). The 3-D Thermal NCA simulation also shows larger clouds at $20 \mathrm{~h}$.

To investigate how cloud size changes over time, we calculated the temporal variation in the autocorrelation length (defined by the shift where the correlation coefficient drops below $1 / e$ ) of the liquid water path of the cloud field (Fig. 14). At about $9 \mathrm{~h}$, the simulations start to develop differently. Both local radiation simulations show an increased correlation length from this time on, indicating larger clouds. The largest clouds are found for the 3-D Thermal NCA simulation, especially towards the end of the period shown here. Both averaged radiation simulations behave quite similarly and show less organization than the local radiation simulations, but slightly more than the No Radiation simulation. The constant cooling simulation is located between the averaged radiation simulations and the No Radiation simulation. A small increase in the correlation length is found at the end of the investigated period for the averaged radiation, which agrees well with Fig. 13.
It shall be mentioned here (although not shown for the above-mentioned reasons) that, from about $24 \mathrm{~h}$ on, large clouds form in the averaged radiation simulations and the constant cooling simulation in which the clouds oscillate: disappearing and then reappearing. No systematic difference between 1-D and 3-D radiation is found in these cases. The local radiation simulations still show cells; however, clouds become larger, especially in the 3-D Thermal NCA simulation.

To further investigate how much water the individual clouds contain and if and how they organize, we show Hovmöller diagrams (Fig. 15). The liquid water path was averaged in $x$ direction for these diagrams. They thus provide an overview of the spatial and temporal development of the cloud field. Extended patches of liquid water along the spatial dimensions indicate large clouds. Extended patches along the time axis show long-living clouds. In addition, the diagrams show how much water is located in the cloud patches. In the No Radiation simulation, no organization occurs. Clouds remain small with little liquid water content throughout the simulation. This is different if thermal radiation is accounted for. If we compare the five different simulations with radiation, one notices that both local radiation simulations and both horizontally averaged radiation simulations as well as the constant cooling simulation show a similar behavior.

Larger fibers containing more liquid water content are found earlier in the simulations with local thermal radiation. Patches of dry and wet regions form (blue until red vs. white areas). The simulations with the horizontally averaged radiation show the first indications of larger structures at the end of the study period, but they contain less liquid water than the local thermal radiation simulations. Also, no significant difference exists between the 1-D and 3-D averaged radiation simulations (which was also evident in Fig. 14). Com- 
(a)
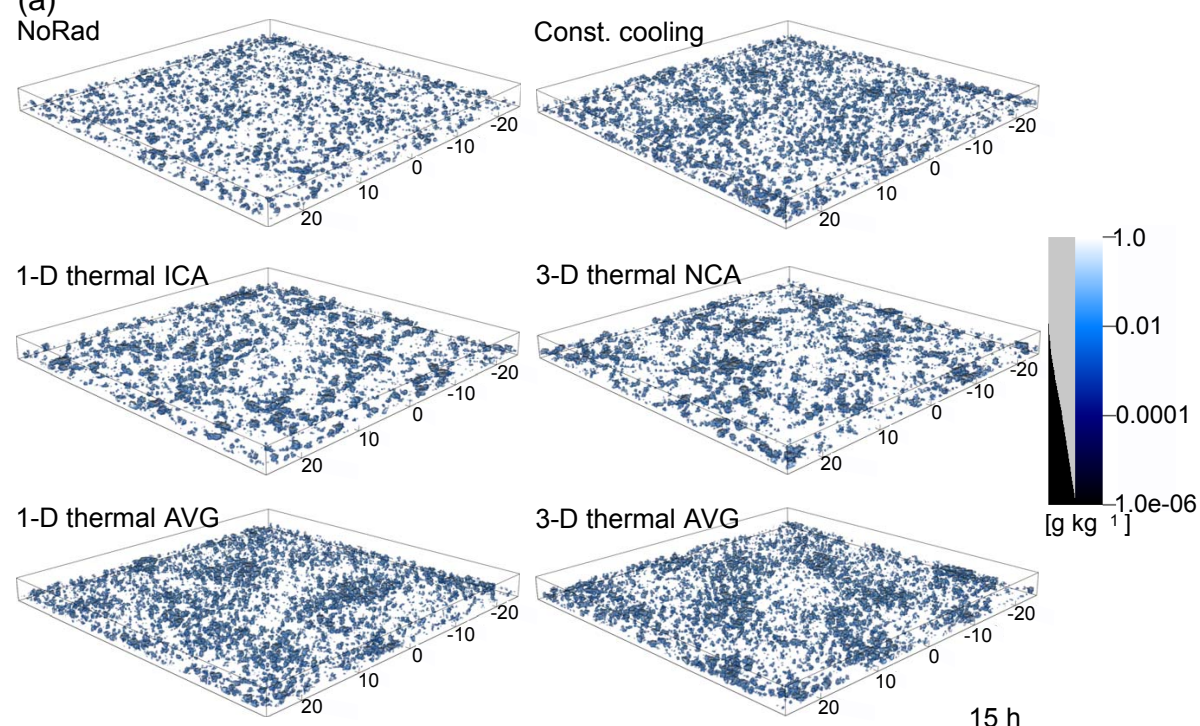

(b)
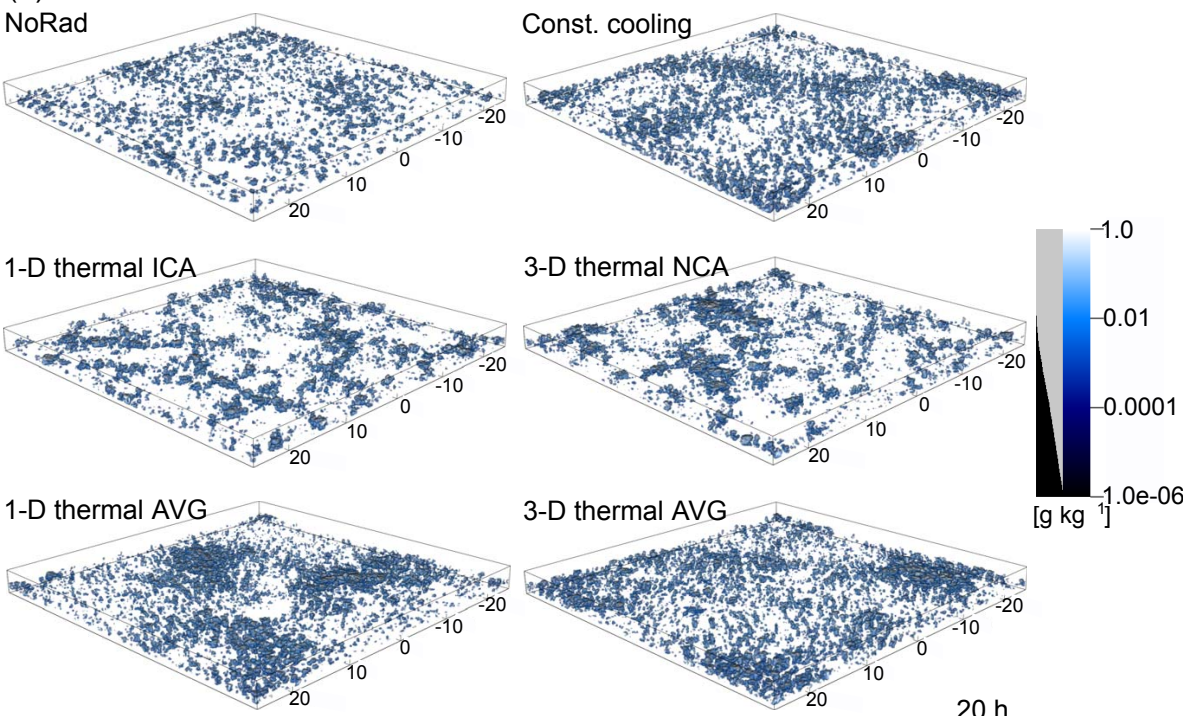

Figure 13. Cloud fields at $15 \mathrm{~h}$ (a) and $20 \mathrm{~h} \mathrm{(b)}$ of the $50 \mathrm{~m}$ resolution simulation. The quantity shown is liquid water mixing ratio. The black and gray bar shows the opacity used in this visualization.

paring the 1-D and 3-D local thermal radiation simulations, we find at the end of the shown period larger structures in the 3-D Thermal NCA radiation simulation. The fiber-like structures of the Hovmöller diagrams usually give a hint about the movement of the clouds. Here, however, the structures show that clouds move very little during their lifetime and mostly remain at one location, as our simulation is performed without any background mean wind.

We can therefore summarize the following findings: local thermal radiation enhances cloud organization in our simulations in the first $20 \mathrm{~h}$ by forming cell structures and larger clouds, concentrating more liquid water in individual clouds.

\section{Cloud lifetime}

The increase in cloud lifetime which could already be seen in the Hovmöller diagrams is quantified in this section. Figure 16 shows a probability density function (pdf) of cloud lifetime. Each cloud occurring within the first $20 \mathrm{~h}$ of our simulations was tracked and the lifetime was calculated. Local thermal radiation leads to less clouds with a small lifetime but more clouds with a larger lifetime.

The results of cloud size and lifetime agree with the results of the last paragraph concerning the cloud organization. Cloud organization and the size dependence of cloud lifetime are closely related, as smaller clouds dying and larger clouds growing will result in fewer but larger clouds and 


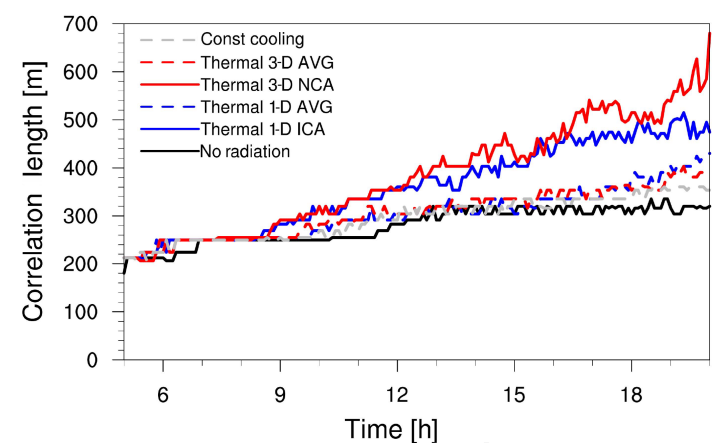

Figure 14. Time development of correlation length. The correlation length is defined by the shift where the correlation coefficient drops below $1 / e$.

in longer correlation lengths. In addition, enhanced turbulence and mixing in the local radiation simulations can lead to a faster decay of small clouds, while larger clouds might live longer and grow due to the enhanced cloud circulation (stronger updrafts and downdrafts). We suspect that, similarly to the single cloud experiment, local thermal radiation reduces cloud diameter and therefore reduces small-size clouds in our simulations. The averaged radiation simulations show more clouds with a longer lifetime than the No Radiation and the constant cooling case.

Figure 17 shows the pdf compared to simulation time and the cloud size. Many small clouds occur at the beginning of the simulations. In the local radiation simulations, these small clouds become fewer over time and more larger clouds occur, while in all other simulations, these small clouds occur throughout the whole simulation. Clouds larger than $1 \times 10^{3} \mathrm{~km}^{2}$ occur in the local radiation simulations, but hardly any in the No Radiation and the constant cooling case.

\section{Summary of cloud development}

In this section, we investigated the effects of different radiation application on the development of a shallow cumulus cloud field. Summarizing, we found that there is a definite difference between a No Radiation and thermal radiation application, where thermal radiation causes more condensation, deeper and more clouds and stronger up- and downdrafts. The boundary layer and cloud layer become deeper and more mixing from the aloft inversion layer occurs. The averaged and the local radiation simulations differ in terms of cloud size, number of clouds and the organization of the cloud field. We find cell structures and larger clouds in the local radiation simulation until $20 \mathrm{~h}$. Simulations with 3-D local radiation develop larger clouds.

In that context, it is not relevant for the organization of the cloud field if the averaged radiation was calculated from the 3-D or the 1-D simulations or if even a prescribed cooling of $2.6 \mathrm{~K} \mathrm{day}^{-1}$ was applied, similar to the findings of Cole et al. (2005).
When we consider local radiation, the differences between 1-D and 3-D thermal radiation are also not large, but we see a tendency at the end of our $20 \mathrm{~h}$ time period for the formation of larger clouds. This is a similar result to Mechem et al. (2008) where differences between 1-D and 3-D thermal radiative transfer in a shallow cumulus cloud field were small as well. We will address the issue of these small differences between the 1-D and 3-D local radiation simulations in Sect. 3.2.4 again.

The main difference between the averaged and the local radiation is the location and the strength of the thermal cooling (as shown in Fig. 18, which summarizes the results of this subsection). In the case of local radiation, the cooling (or heating) acts locally at the cloud sides, tops and bottom. Cooling rates can be as large as several $100 \mathrm{Kday}^{-1}$. This causes a local destabilizing. This is supported by stronger updrafts and downdrafts for the local radiation simulations. The stronger entrainment caused by cloud side cooling shrinks clouds. At the same time, the stronger updrafts lead to stronger cloud growth. It is possible that these two processes vary with the perimeter to area ratio of updrafts, and so the first process can be expected to be successful for small clouds and the second one for large clouds.

In the case of averaged radiation, the resulting cooling is weaker but acts in the entire modeling domain and the cooling does not distinguish between cloudy and cloudless regions. It therefore takes longer for the averaged radiation to destabilize the atmosphere where clouds are located. At the same time, clear sky regions are more destabilized, promoting new development of clouds. This can explain why more and smaller clouds are found during the first $20 \mathrm{~h}$ of the simulations. However, when a certain destabilization is reached, it causes a rapid cloud development in the entire domain at once (in our simulations after $24 \mathrm{~h}$ ).

\subsubsection{Dependence of the results on resolution and reproducibility}

One important issue of our simulations is the robustness of the results and the dependence on resolution. We therefore repeated the calculation with a horizontal resolution of $100 \mathrm{~m}$ instead of $50 \mathrm{~m}$ and performed three runs for the computationally cheaper $100 \mathrm{~m}$ resolution. Although some differences occur between the three $100 \mathrm{~m}$ simulations (one reason being, for example, the randomly chosen spectral bands in the MCSI), the effects (e.g., stronger organization or the more locally focused liquid water in the 3-D local radiation case) remain and are even stronger. This is at first a counterintuitive result, because radiation effects and the 3-D radiation effects are expected to be stronger with better model resolution. We will address this issue in the next subsection (Sect. 3.2.4).

We now focus on some aspects of the $100 \mathrm{~m}$ resolution simulation. Figure 19 shows the time series of cloud fraction and maximum liquid water mixing ratio. In this figure, 

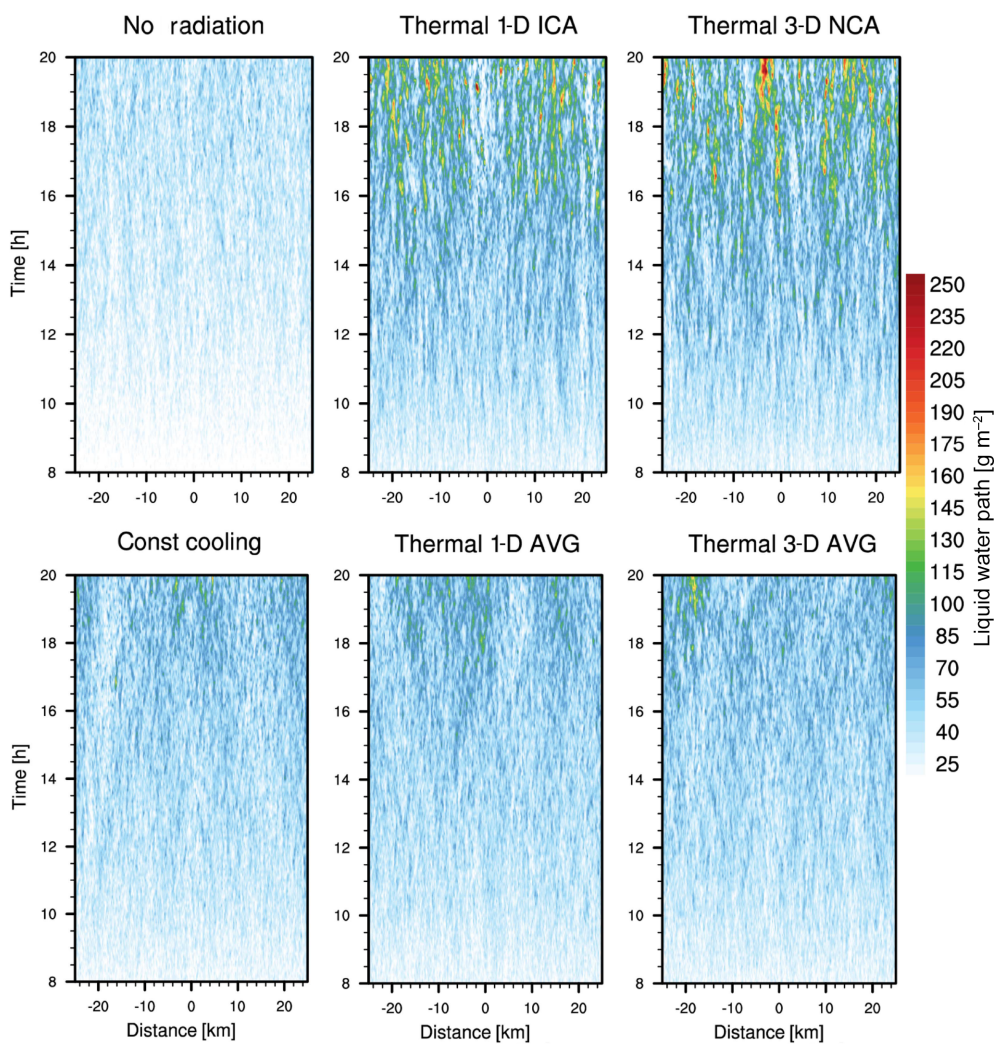

Figure 15. Hovmöller diagram of the liquid water path, averaged in $x$ direction.

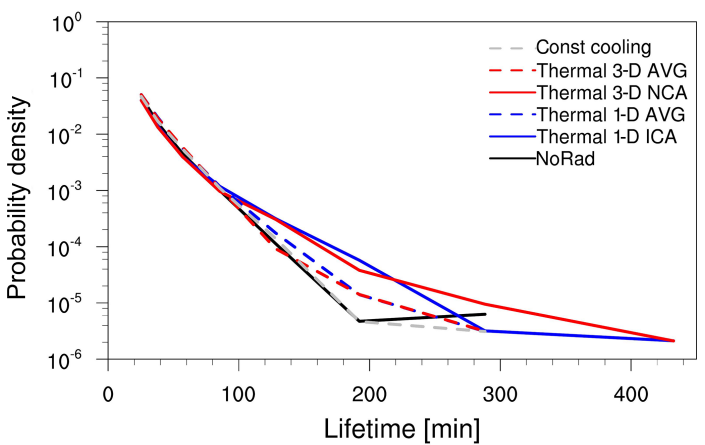

Figure 16. Probability density function of cloud lifetime: the lifetime of each cloud detected by the tracking algorithm was calculated within the first $20 \mathrm{~h}$ of the simulations.

we also show the results of the additional two simulations (thinner lines). Similar to the $50 \mathrm{~m}$ resolution simulations, cloud cover is largest for the averaged radiation simulations. The difference between the 1-D and 3-D radiation simulations, both for local and averaged radiation are larger than in the $50 \mathrm{~m}$ resolution simulations. In terms of liquid water, the same development is found in the $100 \mathrm{~m}$ resolution simulation as in the $50 \mathrm{~m}$ resolution simulation. 3-D effects are also stronger here. The liquid water path and vertical velocity (not shown) show slightly stronger 3-D effects in the $100 \mathrm{~m}$ reso- lution simulations than in the $50 \mathrm{~m}$ resolution simulations, while the difference in cloud base and height remains the same.

Concerning the organization, we start again with snapshots at 15 and $20 \mathrm{~h}$ of the $100 \mathrm{~m}$ resolution simulation, shown in Fig. 20. As might be assumed from the time series, differences between 1-D and 3-D local radiation are larger than in the $50 \mathrm{~m}$ resolution simulation. At $15 \mathrm{~h}$, we find first separation in cloud-free regions and regions of deeper clouds with larger clouds in the 3-D Thermal NCA radiation case. The average radiation simulations show, similarly to the $50 \mathrm{~m}$ simulations, a rather equal distribution of small clouds. At $20 \mathrm{~h}$, we find the cell structures again but only in the 1-D Thermal ICA radiation case. In the 3-D Thermal NCA radiation case, clouds have formed one large patch (if we account for the periodic boundary conditions of the simulation). To account for the whole modeling period, Figs. 21 and 22 show the Hovmöller diagrams and autocorrelation length, this time calculated from the $100 \mathrm{~m}$ resolution simulations. We find larger areas covered by clouds and, at the same time, larger (drier) regions where no clouds form. The clouds of the local radiation simulations also contain more liquid water. The stronger development in the 3-D local radiation case is evident. 

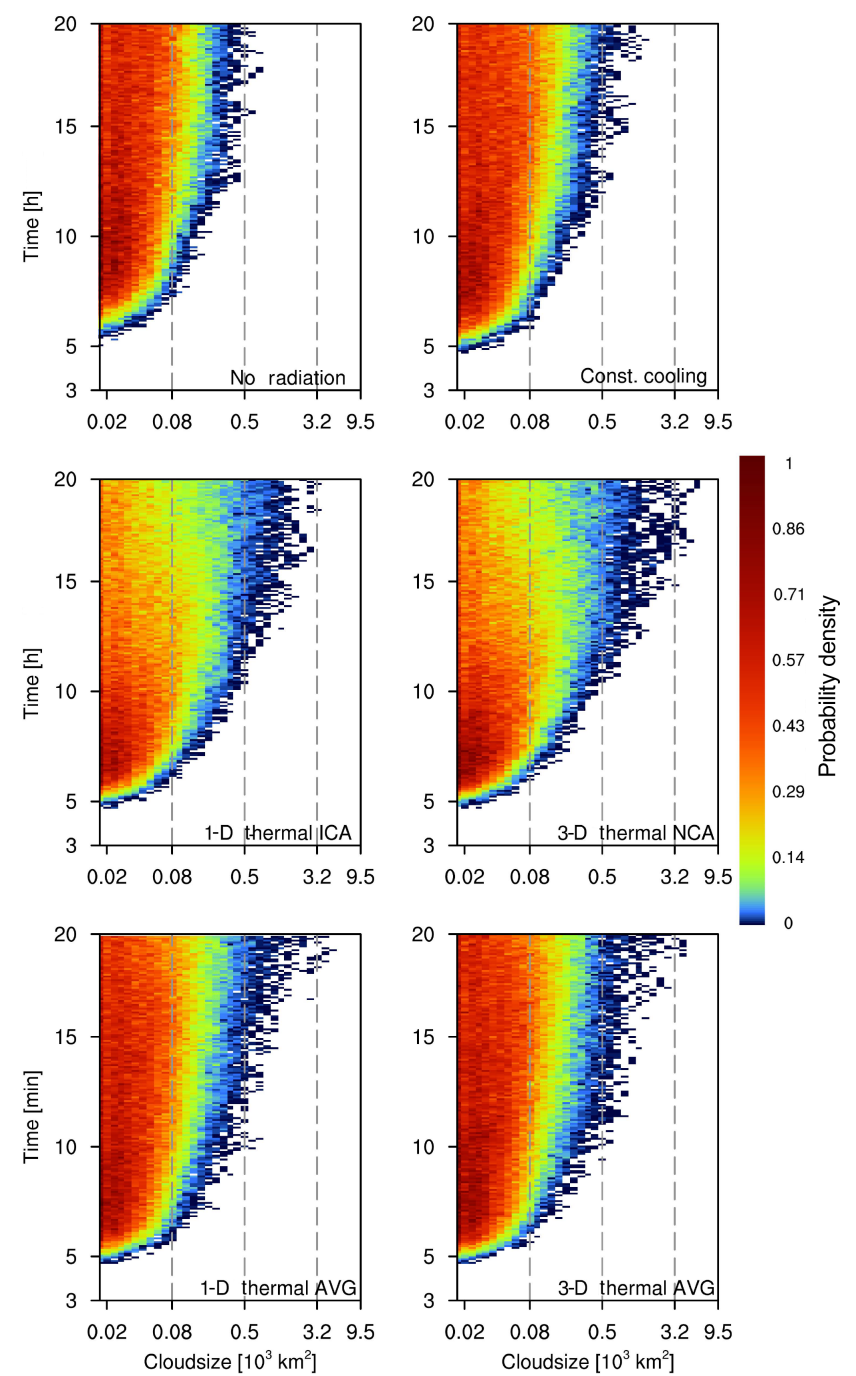

Figure 17. Probability density with respect to cloud size and the time of occurrence in the different simulations.

\subsubsection{Difference in the 100 and $50 \mathrm{~m}$ resolution simulations and the performance of the NCA}

The difference in the development in our simulation and the rather surprising result that 3-D effects are stronger in the $100 \mathrm{~m}$ resolution simulation than in the $50 \mathrm{~m}$ resolution simulation can be related to the limitations of the Neighboring Column Approximation.

The fact that the NCA uses only the direct neighboring column of a grid box to estimate the 3-D cloud side cooling has two implications: first, the "warming" effect of clouds nearby is neglected, which leads occasionally to slightly too high cooling rates. Second, the cloud side cooling is located at the outermost grid box of a cloud. As most of the cloud side cooling is located within the first $50 \mathrm{~m}$ of the cloud, this is still a reasonable assumption if the model resolution is not higher than $50 \mathrm{~m}$. However, if clouds are thin in terms of opti-
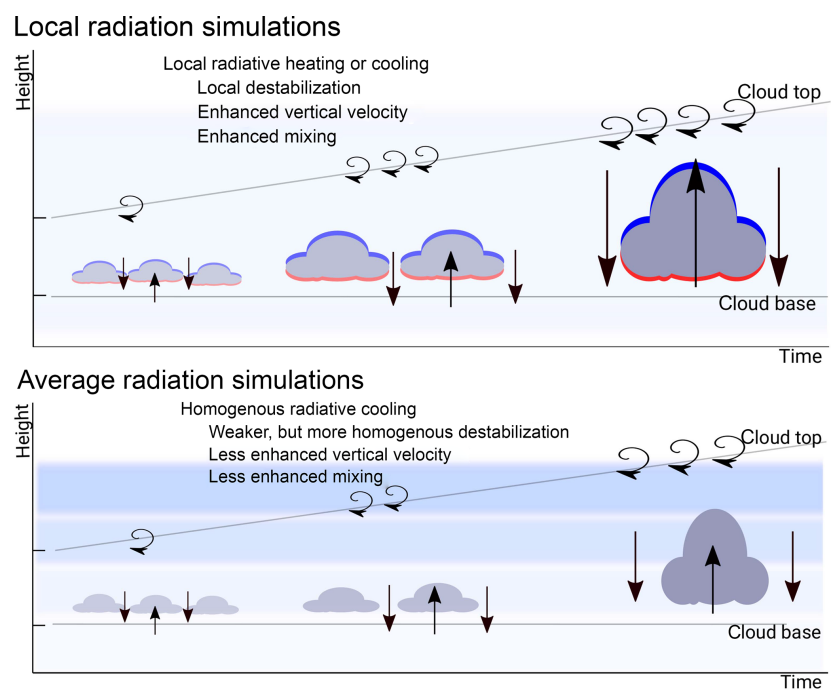

Figure 18. Schematic figure of the effects of thermal radiation in the presented simulations. The figure summarizes cloud development over time and height, showing the enhanced cloud growth, the development of vertical velocity (arrows), a deepening of the cloud layer and the enhanced mixing. Blue and red colors show thermal heating and cooling, either in the cloud itself (for local radiation) or averaged in the cloud layer.

cal thickness, the NCA misses some of the cooling which is, in a real 3-D thermal radiation simulation, found further inside the cloud. As pointed out by Klinger and Mayer (2016), the uncertainty increases for horizontal grid box sizes of $50 \mathrm{~m}$ or smaller. By performing simulations at $50 \mathrm{~m}$ horizontal resolution, we push the NCA to its limits.

Looking into the data of our simulations of this study, we separated the number of cloud side grid boxes and cloud top grid boxes per total number of cloudy grid boxes at three time steps of our simulations $(5,10$ and $20 \mathrm{~h})$. As we increase horizontal resolution only, we expect a factor of 4 more cloudy grid boxes in the $50 \mathrm{~m}$ resolution simulations (assuming that the total cloud volume remains the same). At the same time, the number of cloud side grid boxes increases only by a factor of 2, the number of cloud top grid boxes increases, similarly to the total number, by a factor of 4 . It therefore follows that we find less cloud side grid boxes (per total number of cloudy grid boxes) in the $50 \mathrm{~m}$ resolution simulations. If the optical thickness of those cloud side grid boxes is small, we will therefore neglect some of the 3-D cooling further inside the clouds in the $50 \mathrm{~m}$ resolution simulation.

To see if this is the case in our simulations, we extracted the number of cloud side grid boxes of an optical thickness $<1$ in our cloud data. In the $50 \mathrm{~m}$ resolution simulations, about $30 \%$ of the cloud side grid boxes at $5 \mathrm{~h}$ of simulation have an optical thickness lower than 1 while we find only $12-14 \%$ in the $100 \mathrm{~m}$ resolution simulation. In addition, the number of cloud side grid boxes at $50 \mathrm{~m}$ resolution is less 

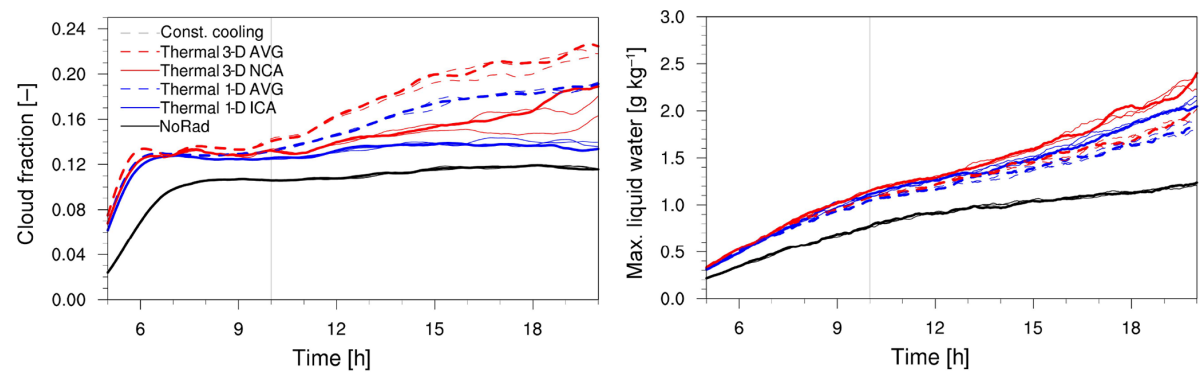

Figure 19. Time development of cloud fraction and maximum liquid water mixing ratio from 5 to $20 \mathrm{~h}$. Thin lines show the results of two additional simulations, respectively.

(a)
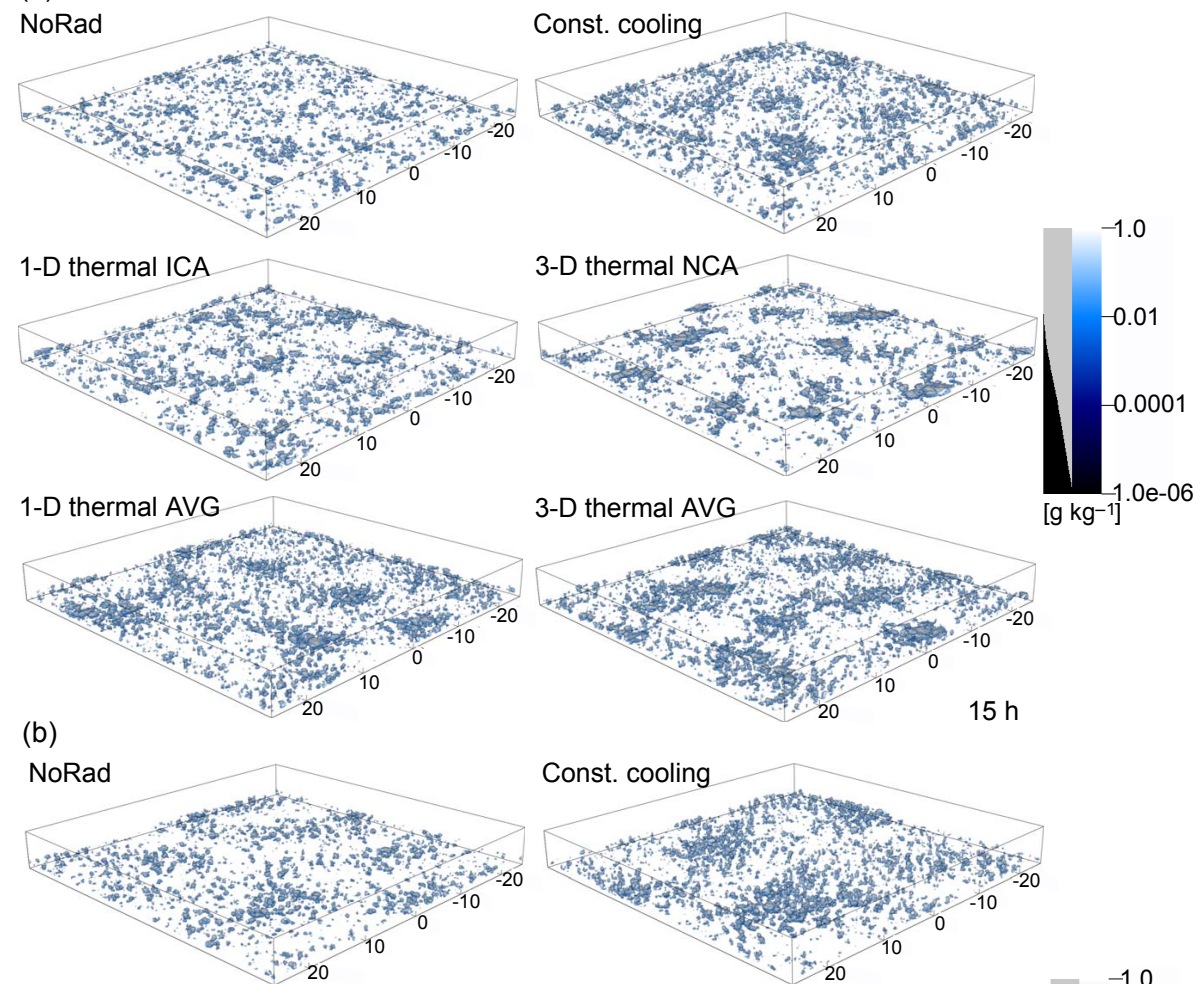

Const. cooling
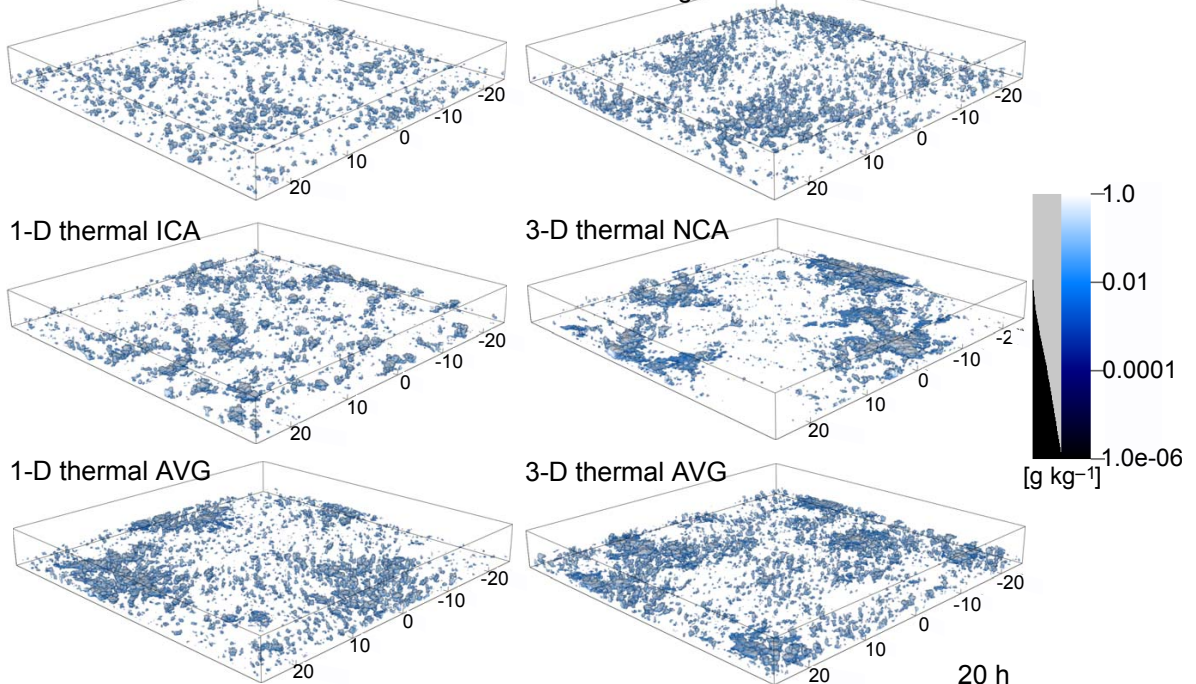

Figure 20. Cloud fields at $15 \mathrm{~h}$ (a) and $20 \mathrm{~h}$ (b) of the $100 \mathrm{~m}$ resolution simulation. The quantity shown is liquid water mixing ratio. The black and gray bar shows the opacity used in this visualization. 

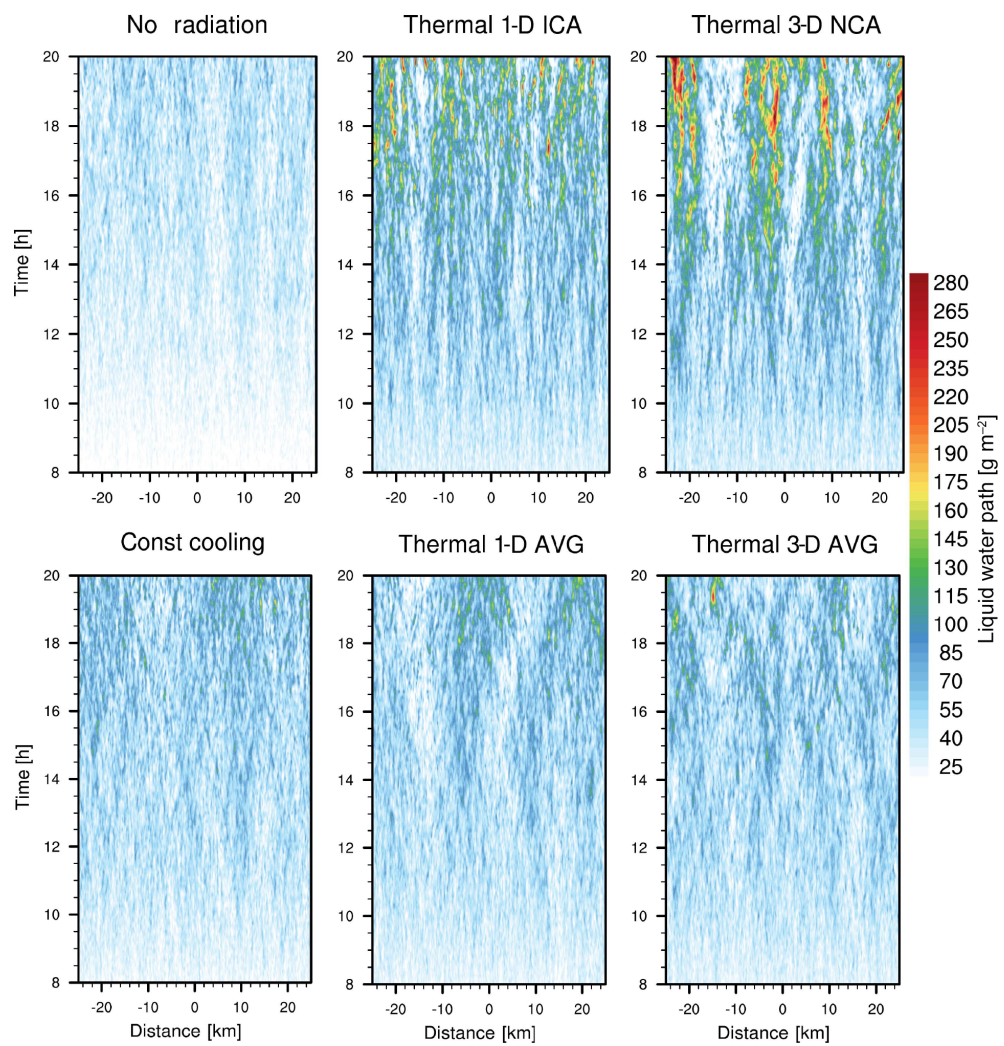

Figure 21. Cloud organization in the $100 \mathrm{~m}$ resolution simulations shown as Hovmöller diagrams of the liquid water path, averaged in $x$ direction.

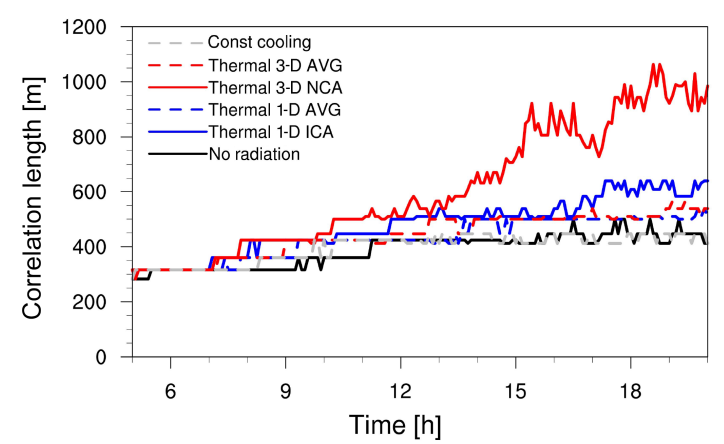

Figure 22. Temporal development of the correlation length for the $100 \mathrm{~m}$ resolution simulation. The correlation length is defined by the shift where the correlation coefficient drops below $1 / e$.

(at $5 \mathrm{~h}$ about $5 \%$ in the $50 \mathrm{~m}$ resolution simulation and about $20 \%$ in the $100 \mathrm{~m}$ resolution simulation).

Therefore, we neglect some of the 3-D cooling further inside the clouds in the early part of our simulations. After clouds have grown and contain more liquid water, the NCA performs better in the $50 \mathrm{~m}$ resolution simulation as well. The exact data from our simulations are shown in Table 3.

We hypothesize therefore that the 3-D cooling is, in an average sense, better calculated in the $100 \mathrm{~m}$ resolution sim- ulations; 3-D effects are stronger there or are evident earlier. In the $50 \mathrm{~m}$ resolution simulations, 3-D effects are (due to the limitations of the NCA) weaker and closer to the 1-D radiation simulations, which explains the smaller differences between 1-D and 3-D thermal radiation at $50 \mathrm{~m}$ resolution.

Although some of the 3-D cooling is missing in the beginning of the $50 \mathrm{~m}$ resolution simulation, we decided to show the 50 and $100 \mathrm{~m}$ resolutions in comparison. While the $100 \mathrm{~m}$ resolution simulation represents the $3-\mathrm{D}$ cooling (on average) better, the $50 \mathrm{~m}$ resolution simulation resolves small-scale structures (as, e.g., the subsiding shell) better.

\section{Conclusions}

We quantified the effect of thermal radiation on cloud development, comparing different radiation approximations. This was first investigated for idealized single clouds induced by a heat bubble perturbation close to the surface as well as for idealized simulations of a shallow cumulus cloud field. Thermal radiation changes the cloud circulation by causing stronger updrafts and stronger subsiding shells around the clouds, which confirms previous results of Guan et al. (1997). However, we extended our study to a comparison of 1-D and 3-D thermal radiation and simulations of a shallow cumulus cloud field. Overall, we find increased mixing and en- 
Table 3. Percentage fraction of cloud side and cloud top grid boxes per total number of cloudy grid boxes at 5,10 and $20 \mathrm{~h}$ of the 50 and $100 \mathrm{~m}$ simulations as well as the percentage fraction of cloud side grid boxes with an optical thickness (at $550 \mathrm{~nm}$ ) lower than 1 .

\begin{tabular}{llccccccc}
\hline \multirow{2}{*}{ Part of cloud } & Resolution & \multicolumn{3}{c}{$50 \mathrm{~m}$} & \multicolumn{3}{c}{$100 \mathrm{~m}$} \\
\cline { 2 - 8 } Cloud top fraction & Simulation time & $5 \mathrm{~h}$ & $10 \mathrm{~h}$ & $20 \mathrm{~h}$ & $5 \mathrm{~h}$ & $10 \mathrm{~h}$ & $20 \mathrm{~h}$ \\
& NoRad & 28 & 22 & 18 & 29 & 19 & 16 \\
& 1-D ICA & 31 & 18 & 15 & 30 & 15 & 13 \\
& 1-D AVG & 31 & 18 & 16 & 29 & 16 & 14 \\
& 3-D NCA & 30 & 18 & 16 & 29 & 16 & 14 \\
& 3-D AVG & 31 & 18 & 14 & 30 & 15 & 13 \\
& Const. cooling & 31 & 19 & 16 & 30 & 16 & 14 \\
\hline \multirow{2}{*}{ Cloud side fraction } & NoRad & 3 & 21 & 20 & 10 & 42 & 40 \\
& 1-D ICA & 6 & 23 & 19 & 18 & 43 & 36 \\
& 1-D AVG & 6 & 21 & 20 & 19 & 46 & 36 \\
& 3-D NCA & 6 & 24 & 18 & 20 & 47 & 36 \\
& 3-D AVG & 6 & 22 & 21 & 20 & 45 & 24 \\
& Const. cooling & 5 & 24 & 23 & 16 & 46 & 44 \\
\hline Cloud side $\tau<1$ & NoRad & 42 & 16 & 13 & 18 & 5 & 5 \\
& 1-D ICA & 31 & 13 & 10 & 12 & 5 & 4 \\
& 1-D AVG & 32 & 14 & 11 & 13 & 5 & 4 \\
& 3-D NCA & 29 & 13 & 10 & 12 & 5 & 5 \\
& 3-D AVG & 31 & 14 & 11 & 12 & 4 & 4 \\
& Const. cooling & 33 & 14 & 11 & 14 & 6 & 4 \\
\hline
\end{tabular}

trainment in the simulations with radiation. Both the mixing and the resulting vertical velocities are due to a destabilization of the atmosphere which results from thermal cooling as, e.g., also found by Sommerai (1976), Fu et al. (1995), Petters et al. (2012) and Lilly (1988). Clouds also become deeper in vertical extent and contain more liquid water if thermal radiation is accounted for.

One important objective of our simulations was to investigate the effect of 3-D local thermal radiation. Therefore, we performed five different thermal radiation simulations. We separate between 1-D and 3-D thermal radiation as well as between local and averaged radiation and a constant cooling simulation. We find that the effects described above are stronger if 3-D thermal radiation is applied, compared to 1-D thermal radiation. The most pronounced difference between the averaged and local radiation simulations and also between the 1-D and 3-D local radiation simulation is the different organization of the cloud field. In a local application of thermal radiation, clouds first organize in cell structures, similarly to those generated with cold pool dynamics. However, as rain is switched off in our simulations, a different process is responsible for this cell development. How these cells really form will be studied in a next step. In the case of local 3-D thermal radiation, we find larger clouds at the end of our simulations. In the $100 \mathrm{~m}$ resolution simulations, we even find a single large cloud patch. The formation of cells is only found in the local radiation simulations; the larger clouds are found in the 3-D local application. Simply adding more cooling to a simulation (as it is done in the averaged ra- diation simulations) does not at all reproduce the 3-D results. The local additional cooling at the cloud side leads to the stronger subsiding shell and the different spatial distribution of the cloud field.

Obviously, the simulations shown in this study are in an idealized framework to omit feedback mechanisms which would occur otherwise. The fixed surface fluxes omit the surface flux feedback which was found, e.g., by Muller and Held (2012) who proposed that this could be a reason for the organization of clouds. Another feedback mechanism which we neglect is the effect of rain and possible cold pool dynamics. Cold pool dynamics are usually associated with cloud organization (e.g., Seifert and Heus, 2013). This cannot be the cause for the cloud organization which we find in our simulations. However, it is obvious that the clouds produced in our simulations are deep enough to cause rain from about $20 \mathrm{~h}$ on, if we would allow it. If we would account for rain effects, the whole system would possibly change. Rain would set in earlier in all thermal radiation simulations (compared to the No Radiation case) and most likely earlier in the local radiation simulations. We rerun the 1-D ICA local radiation simulations, allowing for rain and found rain to occur after $22 \mathrm{~h}$.

Previous studies (Muller and Held, 2012; Muller and Bony, 2015; Emanuel et al., 2014; Wing and Emanuel, 2014) used radiative convective equilibrium (RCE) experiments and found thermal radiation to be a key driver for cloud organization. Our simulations are for a much smaller domain, with higher spatial resolution and without deep convection. 
Yet, we also find that thermal radiation is a driver for organization. Also, our simulations show that it might be essential how radiative transfer is applied. The effects of local and averaged application of radiation differ significantly. While we find differences in the organization of the cloud field in the local 1-D Thermal ICA and 3-D Thermal NCA radiation simulations, the way in which the averaged radiation is applied does not seem to yield significant differences. A similar result was also found by Cole et al. (2005).

Further studies are necessary to show the robustness of the results presented here and an improvement of the 3-D radiative transfer calculations seems to be necessary for highresolution simulations. Additionally, future studies have to be extended to different cloud types and should also account for 3-D solar radiative effects and different feedback mechanisms such as rain, adjusting surface fluxes and more.

Data availability. The UCLA-LES model is publicly available at https://github.com/uclales. Input files and the model code for reproducing the simulations and data of this study are available from the corresponding author upon request.

Competing interests. The authors declare that they have no conflict of interest.

Acknowledgements. This work was funded by the Federal Ministry of Education and Research (BMBF) through the High Definition Clouds and Precipitation for Climate Prediction $\left(\mathrm{HD}(\mathrm{CP})^{2}\right)$ project (FKZ: 01LK1208A), HD(CP) ${ }^{2}$ phase 2 (FKZ: 01LK1504D) and the DFG Transregio 165 "Waves to Weather". We thank George Craig for his very helpful suggestions for the setup of the model experiments and the interpretation of the data. Many thanks go to DKRZ, Hamburg, for providing us with the computational resources. Lastly, we thanks three anonymous reviewers whose comments helped us improve our manuscript.

Edited by: J.-Y. C. Chiu

Reviewed by: three anonymous referees

\section{References}

Bellon, G. and Geoffroy, O.: Stratocumulus radiative effect, multiple equilibria of the well-mixed boundary layer and transition to shallow convection, Q. J. Roy. Meteorol. Soc., 142, 1685-1696, doi:10.1002/qj.2762, 2016a.

Bellon, G. and Geoffroy, O.: How finely do we need to represent the stratocumulus radiative effect?, Q. J. Roy. Meteorol. Soc., 142, 2347-2358, doi:10.1002/qj.2828, 2016 b.

Bony, S., Stevens, B., Frierson, D. M. W., Jakob, C., Kageyama, M., Pincus, R., Shepherd, T. G., Sherwood, S. C., Siebesma, A. P., Sobel, A. H., Watanabe, M., and Webb, M. J.: Clouds, circulation and climate sensitivity, Nat. Geosci., 8, 261-268, 2015.
Boucher, O., Randall, D., Artaxo, P., Bretherton, C., Feingold, G., Forster, P., Kerminen, V.-M., Kondo, Y., Liao, H., Lohmann, U., Rasch, P., Satheesh, S., Sherwood, S., Stevens, B., and Zhang, X.: Clouds and aerosols, in: Climate Change 2013: The Physical Science Basis. Contribution of Working Group I to the Fifth Assessment Report of the Intergovernmental Panel on Climate Change, Cambridge University Press, Cambridge, 571-657, 2013.

Brewster, M.: Evaporation and condensation of water mist/cloud droplets with thermal radiation, Int. J. Heat Mass Trans., 88, 695712, doi:10.1016/j.ijheatmasstransfer.2015.03.055, 2015.

Cahalan, R., Oreopoulos, L., Marshak, A., Evans, K., Davis, A., Pincus, R., Yetzer, K., Mayer, B., Davies, R., Ackerman, T. H. W. B., Clothiaux, E., Ellingson, R., Garay, M., Kassianov, E., Kinne, S., Macke, A., O’Hirok, W., Partain, P., Prigarin, S., Rublev, A., Stephens, G., Szczap, F., Takara, E., Varnai, T., Wen, G., and Zhuraleva, T.: The International Intercomparison of 3D Radiation Codes (I3RC): Bringing together the most advanced radiative transfer tools for cloudy atmospheres, B. Am. Meteorol. Soc., 86, 1275-1293, 2005.

Cheng, A., Xu, K.-M., and Stevens, B.: Effects of Resolution on the Simulation of Boundary-layer Clouds and the Partition of Kinetic Energy to Subgrid Scales, J. Adv. Model. Earth Syst., 2, 3, doi:10.3894/JAMES.2010.2.3, 2010.

Cole, J. N. ., Barker, H. W., Randall, D. A., Khairoutdinov, M. F., and Clothiaux, E. E.: Global consequences of interactions between clouds and radiation at scales unresolved by global climate models, Geophys. Res. Lett., 32, 106703, doi:10.1029/2004GL020945, 2005.

Curry, J. A. and Herman, G. F.: Infrared radiative properties of Arctic stratus clouds, J. Clim. Appl. Meteorol., 24, 525-538, 1985.

Davies, R. and Alves, A.: Flux divergence of thermal radiation within stratiform clouds, J. Geophys. Res., 94, 16277-16286, 1989.

de Lozar, A. and Muessle, L.: Long-resident droplets at the stratocumulus top, Atmos. Chem. Phys., 16, 6563-6576, doi:10.5194/acp-16-6563-2016, 2016.

Emanuel, K., Wing, A. A., and Vincent, E. M.: Radiativeconvective instability, J. Adv. Model. Earth Syst., 6, 75-90, doi:10.1002/2013MS000270, 2014.

Evans, K.: The spherical harmonics discrete ordinate method for three-dimensional atmospheric radiative transfer, J. Atmos. Sci., 55, 429-446, 1998.

Feigelson, E. M.: Radiant heat transfer in a cloudy atmosphere, Transl. into English of the publ. "Luchistyi Reploobmen i Oblaka"', Israel Program for Scientific Translations, Leningrad, 1973.

Feigelson, E. M.: Effects of optical properties of clouds on thermal radiation, in: Radiation in a Cloudy Atmsophere, Springer Netherlands, Dordrecht, 221-231, doi:10.1007/978-94009-6443-3_15, 1984.

Frame, J. W. and Markowski, P.: Numerical Simulations of Radiative Cooling beneath the Anvils of Supercell Thunderstorms, Mon. Weather Rev., 138, 3024-3047, 2010.

Frame, J. W. and Markowski, P.: Dynamical Influences of Anvil Shading on Simulated Supercell Thunderstorms, Mon. Weather Rev., 141, 2802-2820, 2013.

Frame, J. W., Petters, J. L., Markowski, P. M., and Harrington, J. Y.: An application of the tilted independent pixel approximation to cumulonimbus environments, Atmos. Res., 91, 127-136, 2009. 
Fu, Q. and Liou, K.: On the correlated k-distribution method for radiative transfer in nonhomogeneous atmospheres, J. Atmos. Sci., 49, 2139-2156, 1992.

$\mathrm{Fu}, \mathrm{Q}$., Krueger, S., and Liou, K.: Interactions of radiation and convection in simulated tropical cloud clusters, J. Atmos. Sci., 52, 1310-1328, 1995.

Guan, H., Davies, R., and Yau, M.: Longwave radiative cooling rates in axially symmetric clouds, J. Geophys. Res., 100, 32133220, 1995.

Guan, H., Yau, M., and Davies, R.: The Effects of Longwave Radiation in a Small Cumulus Cloud, J. Atmos. Sci., 54, 2201-2214, 1997.

Harrington, J., Feingold, G., and Cotton, W.: Radiative Impacts on the Growth of a Population of Drops within Simulated Summertime Arctic Stratus, J. Atmos. Sci., 57, 766-785, 2000.

Harshvardhan and Weinman, J.: Infrared radiative transfer through a regular array of cuboidal clouds, J. Atmos. Sci., 39, 431-439, 1982.

Harshvardhan, Weinman, J., and Davis, R.: Transport of Infrared Radiation in cuboidal clouds, J. Atmos. Sci., 38, 2500-2513, doi:10.1175/1520-0469(1981)038<2500:TOIRIC>2.0.CO;2, 1981.

Hartman, C. and Harrington, J.: Radiative impacts on the growth of drops within simulated marine stratocumulus. Part I: Maximum solar heating, J. Atmos. Sci., 62, 2323-2338, 2005 a.

Hartman, C. and Harrington, J.: Radiative impacts on the growth of drops within simulated marine stratocumulus. Part II: Solar zenith angle variations, J. Atmos. Sci., 62, 2339-2351, 2005 b.

Heus, T. and Jonker, H. J. J.: Subsiding shells around shallow cumulus clouds, J. Atmos. Sci., 65, 1003-1081, 2008.

Jakub, F. and Mayer, B.: A three-dimensional parallel radiative transfer model for atmospheric heating rates for use in cloud resolving models - The TenStream solver, J. Quant. Spectrosc. Ra., 163, 63-71, doi:10.1016/j.jqsrt.2015.05.003, 2015.

Jakub, F. and Mayer, B.: 3-D radiative transfer in large-eddy simulations - experiences coupling the TenStream solver to the UCLALES, Geosci. Model Dev., 9, 1413-1422, doi:10.5194/gmd-91413-2016, 2016.

Jiang, H., Xue, H., Teller, A., Feingold, G., and Levin, Z.: Aerosol effects on the lifetime of shallow cumulus, Geophys. Res. Lett., 33, 114806, doi:10.1029/2006GL026024, 2006.

Kablick, G., Ellingson, R., Takara, E., and Gu, J.: Longwave 3D Benchmarks for Inhomogeneous Clouds and Comparisons with Approximate Methods, J. Climate, 24, 2192-2205, 2011.

Klinger, C. and Mayer, B.: Three-dimensional Monte Carlo calculation of atmospheric thermal heating rates, J. Quant. Spectrosc. Ra., 144, 123-136, doi:10.1016/j.jqsrt.2014.04.009, 2014.

Klinger, C. and Mayer, B.: The Neighboring Column Approximation (NCA) - A fast approach for the calculation of 3D thermal heating rates in cloud resolving models, J. Quant. Spectrosc. Ra., 168, 17-28, doi:10.1016/j.jqsrt.2015.08.020, 2016.

Koračin, D., Isakov, V., and Mendez-Nuñez, L.: A cloud-resolving model with the radiation scheme based on the Monte Carlo method, Atmos. Res., 47-48, 437-459, doi:10.1016/S01698095(98)00033-7, 1998.

Lábó, E. and Geresdi, I.: Study of longwave radiative transfer in stratocumulus clouds by using bin optical properties and bin microphysics scheme, Atmos. Res., 167, 61-76, 2016.
Larson, V. E., Fleishauer, R. P., Kankiewicz, J. A., Reinke, D. L., and Vonder Haar, T. H.: The death of an altocumulus cloud, Geophys. Res. Lett., 28, 2609-2612, 2001.

Lilly, D. K.: Cirrus Outflow Dynamics, J. Atmos. Sci., 45, 1594 1605, doi:10.1175/1520-0469(1988)045<1594:COD>2.0.CO;2, 1988.

Liou, K.-N., Fu, Q., and Ackerman, T.: A simple formulation of the delta-four-stream approximation for radiative transfer parameterizations, J. Atmos. Sci., 45, 1940-1947, 1988.

Markowski, P. and Harrington, J.: A simulation of a supercell thunderstorm with emulated radiative cooling beneath the anvil, J. Atmos. Sci., 62, 2607-2617, 2005.

Marquis, J. and Harrington, J.: Radiative influences on drop and cloud condensation nuclei equilibrium in stratocumulus, J. Geophys. Res., 110, D10205, doi:10.1029/2004JD005401, 2005.

Mayer, B.: Radiative transfer in the cloudy atmosphere, Eur. Phys. J. Confer., 1, 75-99, doi:10.1140/epjconf/e2009-00912-1, 2009.

Mechem, D. B., Kogan, Y. L., Ovtchinnikov, M., Davis, A., Evans, K., and Ellingson, R.: Multi-Dimensional Longwave Forcing of Boundary Layer Cloud Systems, J. Atmos. Sci., 65, 3963-3977, 2008.

Möller, F.: Long-Wave Radiation, American Meteorological Society, Boston, MA, 34-49, doi:10.1007/978-1-940033-70-9_3, 1951.

Muller, C. and Bony, S.: What favors convective aggregation and why?, Geophys. Res. Lett., 42, 5626-5634, 2015.

Muller, C. J. and Held, I. M.: Detailed investigation of the selfaggregation of convection in cloud-resolving simulations, J. Atmos. Sci., 69, 2551-2565, 2012.

O'Hirok, W. and Gautier, C.: The impact of model resolution on differences between independent column approximation and Monte Carlo estimates of shortwave surface irradiance and atmospheric heating rate, J. Atmos. Sci., 62, 2939-2951, 2005.

Park, S., Gentine, P., Schneider, K., and Farge, M.: Coherent Structures in the Boundary and Cloud Layers: Role of Updrafts, Subsiding Shells, and Environmental Subsidence, J. Atmos. Sci., 73, 1789-1814, doi:10.1175/JAS-D-15-0240.1, 2016.

Petch, J. C. and Gray, M. E. B.: Sensitivity studies using a cloudresolving model simulation of the tropical west Pacific, Q. J. Roy. Meteorol. Soc., 127, 2287-2306, doi:10.1002/qj.49712757705, 2001.

Petters, J., Harrington, J., and Clothiaux, E.: Radiative-dynamical feedbacks in low liquid water path stratiform clouds, J. Atmos. Sci., 69, 1498-1512, 2012.

Pincus, R. and Stevens, B.: Monte Carlo spectral integration: A consistent approximation for radiative transfer in large eddy simulations, J. Adv. Model. Earth Syst., 1, 1, doi:10.3894/JAMES.2009.1.1, 2009.

Savic-Jovcic, V. and Stevens, B.: The Structure and Mesoscale Organization of Precipitating Stratocumulus, J. Atmos. Sci., 65, 1587-1605, doi:10.1175/2007JAS2456.1, 2008.

Schumann, U., Dörnbrack, A., and Mayer, B.: Cloud-shadow effects on the structure of the convective boundary layer, Meteorol. Z., 11, 285-294, 2002.

Seifert, A. and Beheng, K. D.: A double-moment parameterization for simulating autoconversion, accretion and self collection, Atmos. Res., 59-60, 256-382, 2001. 
Seifert, A. and Heus, T.: Large-eddy simulation of organized precipitating trade wind cumulus clouds, Atmos. Chem. Phys., 13, 5631-5645, doi:10.5194/acp-13-5631-2013, 2013.

Sharon, T. M., Albrecht, B. A., Jonsson, H. H., Minnis, P., Khaiyer, M. M., van Reken, T. M., Seinfeld, J., and Flagan, R.: Aerosol and Cloud Microphysical Characteristics of Rifts and Gradients in Maritime Stratocumulus Clouds, J. Atmos. Sci., 63, 983-997, doi:10.1175/JAS3667.1,, 2006.

Small, J., Chuang, Y., Feingold, G., and Jiang, H.: Can aerosol decrease cloud lifetime?, Geophys. Res. Lett., 36, L16806, doi:10.1029/2009GL038888, 2009.

Sommerai, G.: Three-dimensional simulation of turbulent processes in an undisturbed trade wind boundary layer, J. Atmos. Sci., 33, 216-241, 1976.

Stephens, G.: Radiation profiles in extended water clouds. I: Theory, J. Atmos. Sci., 35, 2111-2122, 1978.

Stevens, B.: On the growth of layers of non-precipitating cumulus convection, J. Atmos. Sci, 64, 2916-2931, 2007.

Stevens, B., Moeng, C., Ackerman, A., Bretherton, C., Chlond, A., De Roode, S., Edwards, J., Golaz, J., Jiang, H., Khairoutdinov, M., Kirkpatrick, M., Lewellen, D., Lock, A., Muller, F., Stevens, D., Whelan, E., and Zhu, P.: Evaluation of large-eddy simulations via observations of nocturnal marine stratocumulus, Mon. Weather Rev., 133, 1443-1462, 2005.

Tao, W.-K., Simpson, J., Sui, C. H., Ferrier, B., Lang, S., Scala, J., Chou, M. D., and Pickering, K.: Heating, moisture, and water budgets of tropical and midlatitude squall lines: comparisons and sensitivity to longwave radiation, J. Atmos. Sci., 50, 673-690, 1993.

Tao, W.-K., Lang, S., Simpson, J., Sui, C. H., Ferrier, B., and Chou, M. D.: Mechanisms of cloud-radiation interaction in the tropics and midlatitudes, J. Atmos. Sci., 53, 2624-2651, 1996.

Twomey, S.: Radiative effects in California stratus, Beitr. Phys. Atmos., 56, 429-439, 1983. van Zanten, M., Stevens, B., Nuijens, L., Siebesma, A., Ackerman, A., Burnet, F., Cheng, A., Couvreux, F., Jiang, H., Khairoutdinov, M., Kogan, Y., Lewellen, D., Mechem, D., Nakamura, K., Noda, A., Shipway, B., Slawinska, J., Wang, S., and Wyszogrodzki, A.: Controls on precipitation and cloudiness in simulations of tradewind cumulus as observed during RICO, J. Adv. Model. Earth Syst., 3, M06001, doi:10.1029/2011MS000056, 2011.

Wapler, K. and Mayer, B.: A fast method for the three-dimensional calculation of surface irradiance within a cloud resolving model, J. Appl. Meteorol. Clim., 47, 3061-3071, 2008.

Wing, A. A. and Emanuel, K. A.: Physical mechanisms controlling self-aggregation of convection in idealized numerical modeling simulations, J. Adv. Model. Earth Syst., 6, 59-74, doi:10.1002/2013MS000269, 2014.

Wissmeier, U., Buras, R., und Mayer, B.: paNTICA: A Fast 3D Radiative Transfer Scheme to Calculate Surface Solar Irradiance for NWP and LES Models, J. Appl. Meteorol. Clim., 52, 1698-1715, doi:10.1175/JAMC-D-12-0227.1, 2014.

Xiao, H., Gustafson, W. I., and Wang, H.: Impact of subgrid-scale radiative heating variability on the stratocumulus-to-trade cumulus transition in climate models, J. Geophys. Res.-Atmos., 119, 4192-4203, doi:10.1002/2013JD020999, 2014.

$\mathrm{Xu}$, K.-M. and Randall, D. A.: Impact of interactive radiative transfer on the macroscopic behavior of cumulus ensembles. Part II: Mechanisms for cloud-radiation interactions, J. Atmos. Sci., 52, 800-817, 1995.

Xue, H., Feingold, G., and Stevens, B.: Aerosol Effects on Clouds, Precipitation, and the Organization of Shallow Cumulus Convection, J. Atmos. Sci., 65, 392-406, doi:10.1175/2007JAS2428.1, 2008.

Zinner, T., Mannstein, H., and Tafferner, A.: Cb-TRAM: Tracking and monitoring severe convection from onset over rapid development to mature phase using multi-channel Meteosat-8 SEVIRI data, Meteorol. Appl. Phys., 101, 191-210, doi:10.1007/s00703008-0290-y, 2008. 